\title{
Serial and syntactic processing in the visual analysis of multi-digit numbers
}

\author{
Dror Dotan, Ofir Eliahou, and Sharon Cohen \\ Mathematical Thinking Lab, School of Education and School of Neuroscience, Tel Aviv University
}

\begin{abstract}
The visual analysis of letter strings is a separate cognitive process from the analysis of digit strings. Recent studies have hypothesized that these processes are not only separate but also qualitatively different, in that they may encode information specific to numbers or to words. To examine this hypothesis and to shed further light on the visual analysis of numbers, we asked adults to read aloud multi-digit strings presented to them for brief durations. Their performance was better in digits on the number's left side than in digits farther to the right, with better performance in the two outer digits than their neighbors. This indicates the digits were processed serially, from left to right. Visual similarity of digits increased the likelihood of errors, and when a digit migrated to an incorrect position, it was most often to an adjacent location. Interestingly, the positions of 0 and 1 were encoded better than the positions of 2-9, and 2-9 were identified better when they were next to 0 or 1 . To accommodate these findings, we propose a detailed model for the visual analysis of digit strings. The model assumes imperfect digit detectors in which a digit's visual information leaks to adjacent locations, and a compensation mechanism that inhibits this leakage. Crucially, the compensating inhibition is stronger for 0 and 1 than for the digits 2-9, presumably because of the importance of 0 and 1 in the number system. This sensitivity to 0 and 1 makes the visual analyzer specifically adapted to numbers, not words, and may be one of the brain's reasons to implement the visual analysis of numbers and words in two separate cognitive processes.
\end{abstract}

\section{Introduction}

The human ability to combine visually presented letters and digits into words and numbers, to understand them, and to say them aloud is anything but trivial. Most adult readers are able to convert letter strings and digit strings into words and numbers while applying complex conversion rules, and they do so quickly, effortlessly, and with only a few mistakes. Although letters and digits have similar visual characteristics, the visual analysis of letter strings and digit strings is performed by two separate cognitive systems, located in separate brain regions (Abboud et al., 2015; Crutch \& Warrington, 2007; Déjerine, 1892; Priftis et al., 2013; see review in Dotan \& Friedmann, 2019).

\footnotetext{
${ }^{\circ}$ We thank Maya Yachini and Naama Friedmann for their advice on the analysis and interpretation of the data, Tom Maayan for her help in the data processing, and Elizabeth Thompson for reviewing the manuscript. This research was supported by a grant from the Jacobs Foundation (Dotan, 2019-1320-05).
} 
Here we examine the visual analysis of digit strings in detail and ask why it is separate from the that of letter strings.

Dotan and Friedmann (2018) recently proposed a detailed cognitive model for reading aloud digit strings as numbers. In accordance with previous studies (e.g., Benson \& Denckla, 1969; Dehaene, 1992; McCloskey, 1992), this model postulates separate processes for the visual analysis of the digit string and the verbal production of the corresponding number words. The model further proposes that visual analysis consists of 5 sub-processes: encoding the digit identities; encoding their relative order; detecting how many digits the number has; encoding the positions of 0; and grouping the digits into triplets. The first two processes provide the sequence of digits in the number. The latter 3 processes encode the syntactic structure of the digit string (hereafter "decimal structure"). The decimal structure (number length, 0 positions, grouping into triplets) and the ordered digit identities are redundant pieces of information, however, there may be good reasons for the visual analyzer to put extra effort into extracting the decimal-structure information from the digit string via dedicated processes: this information is especially important for the subsequent processing stages (Cohen \& Dehaene, 1991; Dotan \& Friedmann, 2018). In particular, if the task at hand requires converting the number to its verbal representation (e.g., when reading aloud), the digit string's decimal structure is important because it determines the syntactic structure of the number's verbal representation - the number of words to be produced and their lexical classes (ones, tens, etc.; Cohen \& Dehaene, 1991; McCloskey et al., 1986). Thus, reliable encoding of the decimal structure may help create the verbal representation more quickly and with fewer errors. The decimal structure is also important if the task requires converting the number to the quantity it represents - e.g., when deciding whether a number is smaller or larger than another number. In this case, the number length may be required to initialize the digits-to-quantity conversion mechanism (Dotan \& Dehaene, 2020b), and 0 seems to be processed in the quantity system in a different way than other digits (Pinhas \& Tzelgov, 2012).

These particular properties of numbers provide a principled reason for the existence of the 3 syntactic processes within the digit visual analyzer. Importantly, they also provide a possible reason for the cognitive separation of the visual analyzers of digit and letter strings: even if letters and digits are visually similar, the syntactic structures of letter strings and digit strings are quite different. The structure of a digit string is described above; letter strings have structure as well, e.g., the word's morphological structure, the distinction between consonants and vowels etc. Thus, understanding how the numeric visual analyzer handles the number syntax may shed light not only on the number reading mechanisms, but also on the reasons for the distinction between wordparsing and number-parsing processes. The present study focuses on two specific aspects of the visual analysis of numbers: the order of processing the digits and the putative dedicated process that encodes the position of 0 . 


\subsection{The order of processing digits}

Only a few studies have examined the order of processing the digits in a digit string. Most of these have aimed to arbitrate between two models - either processing the digits in parallel or processing them serially from left to right - and used a number-comparison paradigm. In this paradigm, participants see two multi-digit numbers and are asked to judge which is larger, or, alternatively, they see a single number and are asked to compare it to a fixed reference. These studies have concluded that the digits of 2- and 3-digit numbers are processed in parallel, but in longer numbers, they are processed serially (Bahnmueller et al., 2016; Dotan \& Dehaene, 2020b; Meyerhoff et al., 2012).

As far as we know, only one study has examined the order of processing the digits in a reading aloud task (Friedmann et al., 2010). This study described two adults with a reading disorder that selectively impaired their encoding of the relative order of digits in a digit string. These two individuals made many digit transposition errors when reading numbers; crucially, they made more errors for digits farther to the right, with the maximal number of transpositions being between the decade and the unit digits. One explanation of this finding is that the digits were processed serially, from left to right. Several specific architectures can explain why such serial processing could result in the error rate increasing for digits farther to the right; for example, the cognitive load may increase with additional digits, or later digits may receive less attentional resources. Note, however, that serial processing is not the only interpretation of a left-to-right increasing error gradient; an alternative interpretation could be an attentional bias to the left. We return to this point in the Discussion.

Friedmann et al. (2010) clearly showed the error rate was higher for digits farther to the right. However, the two participants in their study had a digit-order encoding deficit, which may have affected the order of processing the digits, so it is hard to generalize any conclusion to the general population. In our study, to assess serial versus parallel processing in number reading, we examined the error-by-position pattern for participants without cognitive disorders. As we shall see, we observed a similar serial-like pattern here too.

In readers without disorders, another effect is sometimes observed: fewer errors in the leftmost and rightmost digits than in the inner digits (Chanceaux \& Grainger, 2012). This outer-character advantage is a common finding in tasks using letter strings (Estes et al., 1976; Gomez et al., 2008; Hammond \& Green, 1982; Townsend et al., 1971; Tydgat \& Grainger, 2009; Wolford, 1975). One

explanation of this effect is crowding. More specifically, the two outer characters have fewer neighboring characters than inner characters, so they are less susceptible to visual interference. Note that the outer-digit advantage is not mutually exclusive to serial or parallel processing - it may exist in both.

Experiments showing an outer-digit advantage have typically used a character-detection task, arguably evoking a visual analysis process that differs somewhat from the process involved in 
reading words and numbers. Still, as we go on to explain, here we observed a similar outer-digit advantage with a number-reading task. In the Discussion, we return to possible interpretations of the outer-digit advantage.

\subsection{Detecting positions of the digit 0}

Dotan and Friedmann (2018) proposed that 3 visual analysis processes encode the digit string's decimal structure: encoding the number length, grouping the digit string into triplets, and encoding the positions of 0 . Of these three processes, for the former two, Dotan and Friedmann showed clear neuropsychological dissociations: they found at least one individual for whom each of the two processes was selectively impaired (participants MA, ED, NL). In contrast, for the "zero detector", they only found a subtle dissociation: participant EY, who had a selective deficit in digit position encoding, made digit position errors in the digits 2-9 but not in 0. Another relevant study was by Cohen and Dehaene (1991). Their patient YM made digit substitution errors in the digits 2-9 but not in 0 and 1. The effect was clear, but YM showed the superiority of 0 and 1 only in some tasks, so this effect could not be unambiguously attributed to the visual analyzer. Thus, although both studies showed 0 is different from the other digits, additional evidence may be useful to unequivocally support the notion of a dedicated zero-detection process within the visual analyzer. In the present study we provide such evidence, and we also examine in more detail how 0 and 1 are processed.

Another issue is the role of the digit 1. The principled reason for a zero-detection process is presumably the special role of 0 in the number system. In the verbal system, 0 is never pronounced when it appears in a multi-digit string. In the quantity system (Approximate Number System, ANS; Dehaene, 1997), zero - "nothing" - is perceived as qualitatively different from the "something" quantities (Pinhas \& Tzelgov, 2012). The role of 1 does not seem to be as special in either system. In the verbal system, the digit 1 creates a verbal irregularity only in the case of teen words (in English as well as in Hebrew, in which the present study was conducted). In the ANS, 1 may be processed like any other quantity. This raises the possibility that the visual analyzer does not treat 1 in a special manner as it treats 0, or at least not to the same extent. Indeed, Dotan and Friedmann (2018) hypothesized that the special treatment of 1 is not a default behavior of the visual analyzer, but occurs only in the context of a reading aloud task. In their study, participants showed superior processing of both 0 and 1 , and the task was reading numbers aloud. In the present study too, our findings suggested that at least when the task is reading numbers aloud, 1 is treated in a special manner.

\subsection{The present study}

Our participants read aloud multi-digit numbers presented as digit strings. Thus, unlike previous studies, we examined serial versus parallel processing in a natural reading context among adults without reading disorders. We examined the errors rates for each digit. Similar to Friedmann 
et al. (2010), we reasoned that if the digits were processed serially, the error rate should increase for digits farther to the right. This was indeed the case. Some of the numbers included 0 and 1; this allowed us to examine whether the processing of these two digits was superior to the processing of the digits 2-9. We found that it was. Our participants did not have any known cognitive disorders, so to elicit errors, we presented each number for a short duration.

\section{Method}

\subsection{Participants}

Twenty-five adults participated in this study. They were native speakers of Hebrew without known cognitive disorders and with normal or corrected-to-normal vision. All participants gave informed consent. One participant was extremely inattentive and was excluded. The remaining 24 participants were 20-63 years old (mean $=40 ; 6, \mathrm{SD}=12 ; 11)$.

\subsection{Stimuli and Procedure}

Each participant read aloud 240 multi-digit numbers presented as digit strings on a computer screen. The numbers were presented in Arial 64 font. Each number appeared for $50 \mathrm{~ms}, 100 \mathrm{~ms}$, or $200 \mathrm{~ms}(79,81$, and 80 items, respectively). The numbers had 3, 4, or 5 digits $(30,80$, and 130 items respectively, distributed equally across exposure durations; Fig. 1). The digit 0 appeared in half of the numbers, and the digit 1 appeared in 40 numbers, distributed equally across exposure durations. Fourteen numbers included both 0 and 1, and 94 numbers included neither, i.e., only the digits 2-9. The participants responded in Hebrew, their native tongue. The verbal number system in Hebrew is similar to English, with minor differences (see Supplementary Material for details).

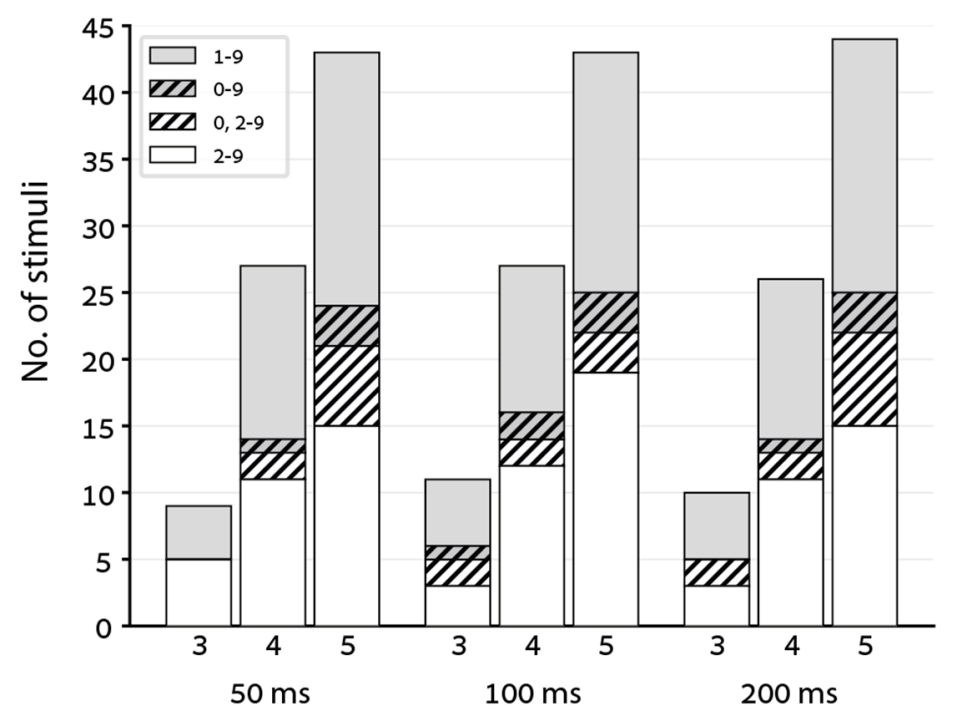

Fig. 1. Distribution of stimuli. In each trial, a number with 3 to 5 digits was presented briefly, and the participant read it aloud. The bars show the distribution of targets (same targets for all participants). Grey 
indicates the number included the digit 1 , and stripes indicate it included 0 . We examined whether the participant produced each target digit in the correct position, in an incorrect position, or not at all.

The 240 stimuli were administered in 5 blocks, with 48 numbers in each block. Participants were instructed to read as accurately as possible and, if they did not know the whole number, to provide partial information; e.g., "it started with 5", “it had four digits", "it included 7, I don't remember where", etc. Their responses were written down immediately by the experimenter and were audio recorded and double-checked by another researcher.

We used three different exposure durations to present the numbers because we hoped this would provide an additional way to examine serial processing. We reasoned that if the digits were processed serially, we might observe good identification of the digits on the left, with an abrupt drop in performance starting from a certain position. As we did not observe such a pattern, we added exposure duration as a covariate, but no analysis specifically focused on it.

The experimental protocol was approved by the Tel Aviv University ethics committee.

\subsection{Error coding}

We coded all the participants' responses, including responses with only partial information about the number (e.g., fifty something). We classified the participants' errors into three types: number-length errors (coded for each multi-digit number), digit identification errors, and digit position errors (the latter two were coded for each digit).

Number length. For each number, we coded whether the participant said a number with the correct number of digits or not. We coded a number-length error when the participant's response implied a number with an incorrect number of digits - e.g., reading 230 as two thousand and thirty, twenty three, or twenty something. Technically, a number length error means the first digit was produced within an incorrect lexical class (ones, tens, hundreds, etc.).

Digit identification. Each target digit was considered identified if the response included this digit in any position. For example, when 320 is read as two hundred and three, all digits are considered identified. Failure to identify a digit may result from substitution (e.g., $34 \rightarrow$ thirty five; or substitution of a digit to zero, i.e., omission of a number word: $234 \rightarrow$ two hundred and four); digit omission $(234 \rightarrow$ twenty four; note that this would be coded as a double error - a digit identification error and a number-length error); not knowing a digit (23 $\rightarrow$ twenty something; 23 $\rightarrow$ it had "2" in it); an "I don't know" response (no digits identified); or a digit overridden by a migrating digit (e.g., the digit 3 in $234 \rightarrow$ two hundred and forty or two hundred and forty four).

Digit position. If the digit was identified, we could examine whether it was produced in the correct position or had a position error. For example, reading 23 as thirty two was coded as a position error for both digits. Reading 23 as thirty something or as thirty five was coded as a position error only for the digit 3 (and an identification error for 2). The digit positions were coded 
relative to the number's right end, i.e., according to the digit's decimal role. However, in the event of a number length error, if the number's first digits maintained their positions relative to the left end of the number, we did not code this as a position error. For example, there are no position errors in reading 1203 as 123 or as 10203. Similarly, in reading 1034 as 143, there are position errors for the digits 3 and 4 but not 1 . We used this coding scheme because number length errors probably originate in a process other than position coding (Dotan \& Friedmann, 2018), and malfunctions of the number-length mechanism should not count as position errors.

Digit accuracy. A response digit was defined as accurate if the digit was identified and produced in the correct position. Accuracy was used only for few specific analyses. In most cases, we analyzed identification and position separately.

Items with multiple responses. Participants sometimes provided more than one number as a response, e.g., reading 23 as twenty five... no, forty three. In such situations, we coded for each digit the errors from all responses given by the participant. In the example above, both digits would count as unidentified: the response twenty five fails to identify the digit 3 , and the response forty three fails to identify the 2 . Nevertheless, if the participant started saying one number and then stopped in order to say a corrected number, the missing digits in the first number did not count as unidentified. For example, reading 123 as one hundred and... one hundred and twenty three is a correct response, with no errors. Reading 123 as one hundred and thirty... one hundred and twenty three would be coded as a position error for the digit 3 (because of the first response) and no identification error.

The participants' responses, our coding of errors for each trial, and the analysis scripts can be found on http://osf.io/2adcs.

\subsection{Statistical analysis}

To examine which factors affected identification errors, we submitted the data to a logistic linear mixed model (LLMM) with the factors in question as predictors. The analysis unit was a digit, and the binary dependent variable was digit identification. These LLMMs usually included factors such as the digit position, number length, exposure duration etc., and they always included the participant as a single random factor. When digit position was included it was always a numeric factor, to account for the assumption of a monotonous left-to-right gradient. When number length and exposure duration were included, they were always categorical (non-numeric) factors, so not to assume a-priori that their effect was linear or monotonous. We used R (R Core Team, 2019) with the lme 4 package (Bates et al., 2015). To determine whether the effect of a particular factor was significant, we first verified that its model coefficient was in the predicted direction. Then, we used a likelihood ratio test to compare the LLMM to an identical model but without the factor in question. For these comparisons, we report the test statistic $2\left(\mathrm{LL}_{0}-\mathrm{LL}_{1}\right)$, which follows a $\chi^{2}$ distribution ( $\mathrm{LL}_{0}$ and $\mathrm{LL}_{1}$ denote the log-likelihoods of the reduced model and the full model), and 
the corresponding $p$ value. The degrees of freedom are not reported as they were always 1 . We also report the odds ratio of the factor in question (the exponent of the factor's coefficient in the full model). In Supplementary Material, we report odds ratios and confidence intervals for all factors in each model.

We used the same method to examine which factors affected position errors, now with correct/incorrect position as the binary dependent variable. The analyses of position errors included only the digits that were identified. As a result, some participants contributed more data points than others to the analysis. To control for a potential bias, we weighted each data point in inverse proportion to the participant's total number of data points, but all results were essentially replicated when we avoided this weighting.

\section{Results}

The task was clearly not easy: the average digit identification rate was $63 \%$ (SD $=13 \%$; the per-participant average was between $36 \%$ and $87 \%$ ). The identified digits were produced in the correct position in $86 \%$ of the cases, on average ( $\mathrm{SD}=5 \%$; the per-participant average was between $78 \%$ and 95\%). Fig. 2 shows participants' performance for each number length and exposure duration.

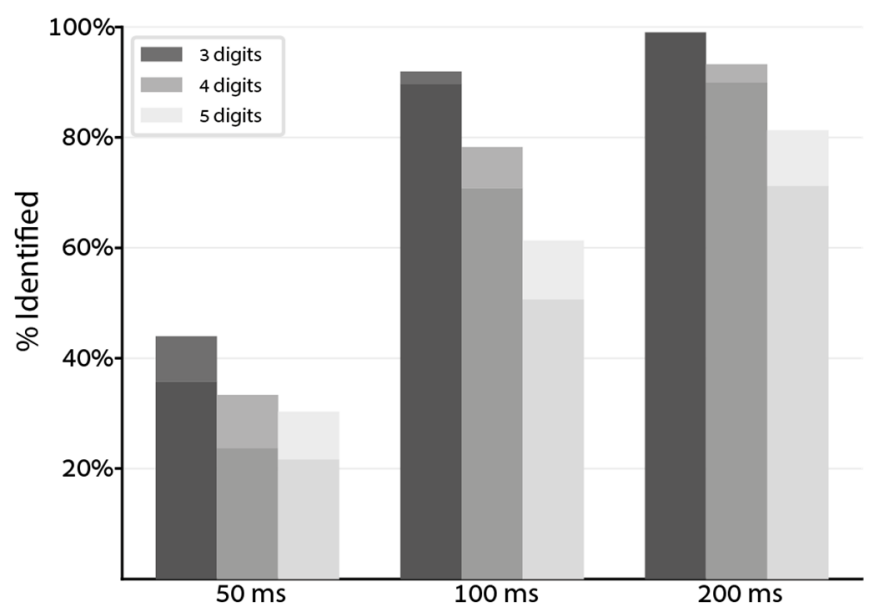

Fig. 2. The rate of identified digits separately for each number length and exposure duration. Each bar shows the rate of digits identified in the correct position (bottom, darker color) or an incorrect (top) position. Unsurprisingly, there were fewer errors in shorter numbers and with longer exposures.

\subsection{Digits 2-9: serial processing or outer-digit advantage?}

\subsubsection{Digit identification}

We start by analyzing the numbers that included neither 0 nor 1 , focusing on digit identification, irrespective of whether the digit was produced in the correct position or not. If digits are processed serially from left to right (serial-processing hypothesis), the identification rate should 
decrease monotonously for positions farther to the right. If the outer digits are processed better than the inner digits (outer-digit advantage hypothesis), the identification rate for the outer digits should be higher than for the inner digits. The results (Fig. 3a) confirmed both predictions. The identification rate decreased for positions farther to the right, as predicted by the serial-processing hypothesis. At the same time, there was an increase in the unit digit relative to the decade digit (at least for exposure $\geq 100 \mathrm{~ms}$ ), as predicted by the outer-digit advantage hypothesis.

We used several methods to show both effects were statistically reliable. First, the digit identification data were submitted to the logistic linear mixed model (LLMM) analysis described in Section 0, with the participant as a random factor and 4 within-subject factors: the digit position as a numeric factor to account for the serial processing hypothesis; a factor indicating whether the digit was outer or inner, to account for the outer-digit advantage hypothesis; the number length (non-numeric); and the exposure duration (non-numeric). The results provided support for both hypotheses, confirming the visual impression given by Fig. 3a. In support of the serial processing hypothesis, the Position factor was significant (odds ratio $=1.84, \chi^{2}=848.0$, $p<.001$; full results in Table S3). In support of the outer-digit advantage hypothesis, the Outer/Inner factor was significant too (odds ratio $=3.47, \chi^{2}=543.7, p<.001$ ). These two factors remained significant when we excluded trials with number-length errors, and when we analyzed each exposure duration and number length separately (for numbers with 4 digits or more, all $p<$ .001 ; 3-digit numbers were excluded because in Fig. 3a they did not show a clear Outer/Inner trend).

We also compared the identification rate in the unit digit versus the decade digit, for which the two hypotheses make opposite predictions: the outer-digit advantage hypothesis predicts better identification of the unit digit, but the serial-processing hypothesis predicts better identification of the decade digit. Numerically, the identification rate was higher for the unit digit, thus supporting the outer-digit advantage hypothesis (Fig. 3a). To examine this effect statistically, we submitted the digit identification data of decades and units to an LLMM (Section 0) with the participant as a random factor and two within-subject non-numeric factors: digit position (decade or unit) and exposure duration. This analysis confirmed the visual impression: the Position factor was significant (odds ratio $=0.61, \chi^{2}=45.7, p<.001$; full results in Table S4). Running the same analysis separately for each number length and then for each exposure duration showed this effect was driven by 5-digit numbers (odds ratio $=0.46, \chi^{2}=63.8, p<.001$; for 4-digit numbers, odds ratio $=0.83, \chi^{2}=2.1, p=.15$ ) and by longer exposure durations (5-digit numbers: at $50 \mathrm{~ms}, \chi^{2}=$ $2.3, p=.13$; at 100 and $200 \mathrm{~ms}$, odds ratio $=0.46$ and 0.30 , respectively; $\chi^{2}>27.8, p<.001$; digit numbers: at 50-100 ms, $\chi^{2}<1.2, p>.27$; at $200 \mathrm{~ms}$, odds ratio $\left.=0.47, \chi^{2}=8.3, p=.004\right)$. To be precise, the advantage of the outer digit (unit) relative to the digit next to it (decade) increased as the number lengthened and as the participant saw it for more time. 
(a) Identification
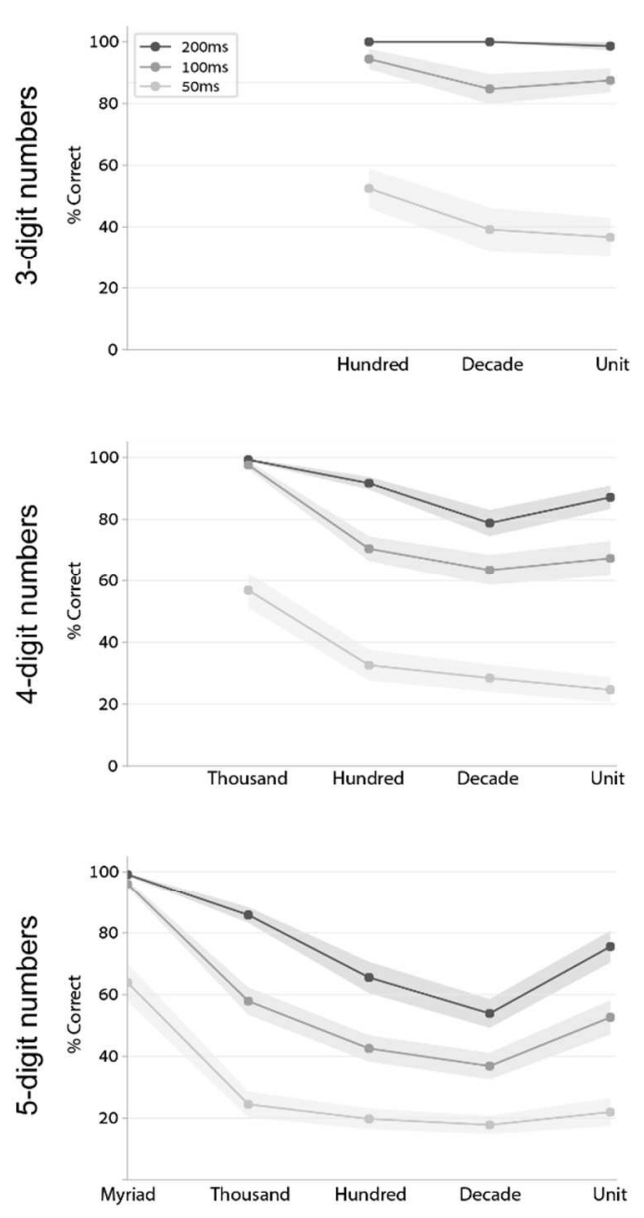

(b) Position
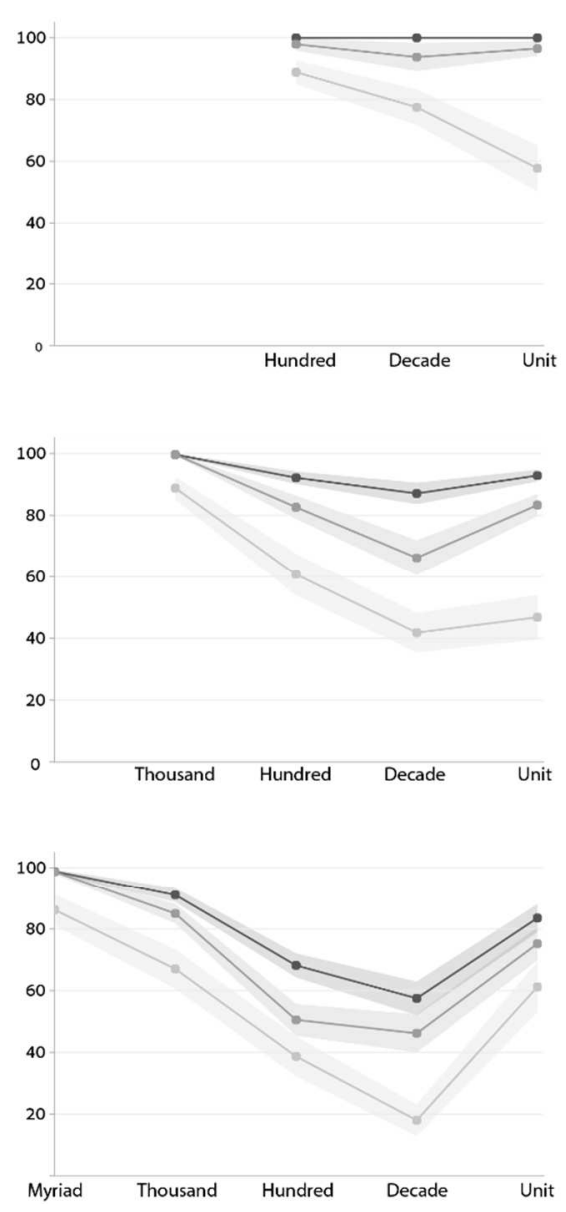

(c) Accuracy
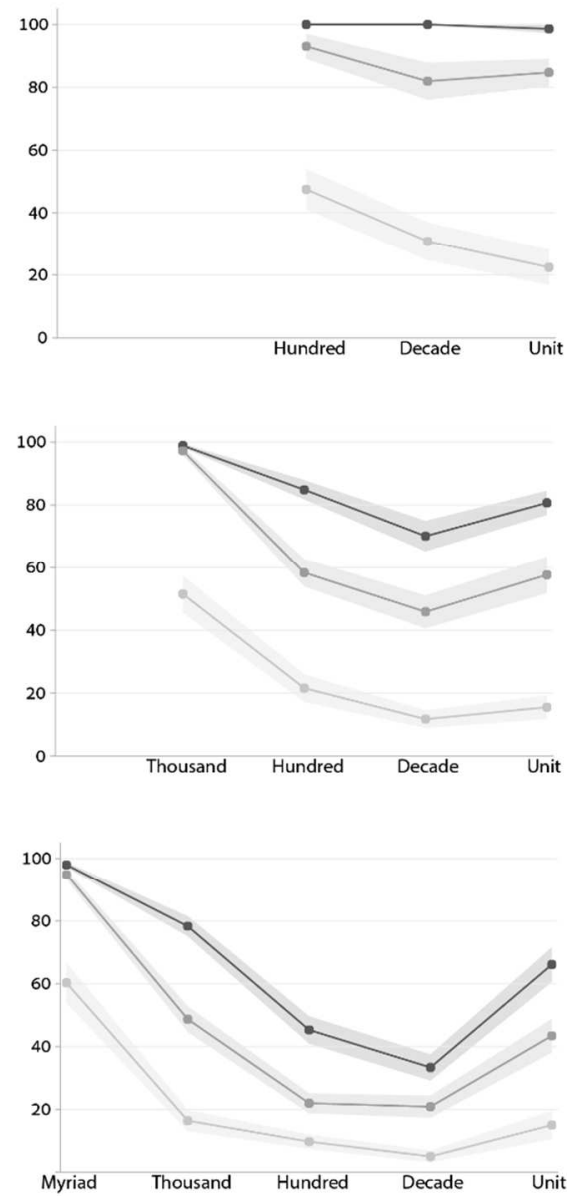

Fig. 3. Performance in each position when reading aloud numbers including only the digits 2-9, for each number length and exposure duration. The left column (a) shows the rate of identified digits. The middle column (b) shows the rate of digits produced in the correct position out of all identified digits. The right column (c) shows the rate of digits identified correctly and in the correct position. For all three measures, the performance decreased for positions farther to the right, in accordance with the assumption of serial left-to-right processing of digits. For 5-digit numbers and for 4-digit numbers with longer exposures, performance in the unit digit was higher than in the decade digit, in accordance with the assumption that outer digits are processed better than inner digits. Essentially the same patterns were observed when the numbers with 0 and 1 were included (Fig. S1).

Additional anecdotal support for an outer-digit advantage comes from some trials in which participants identified a digit and did not know its precise position but knew it was not the first or the last digit. Although this did not happen very often, the participants never explicitly excluded any other position except the first and the last.

If both hypotheses - serial processing and outer-digit advantage - are indeed true, the performance in the first (leftmost) digit should be much better than in the other digits; the leftmost digit is superior both because it is the first and because it is an outer digit. Fig. 3a suggests this was 
indeed the case. The digit identification rate dropped abruptly between the first and the second digits, and although it kept dropping for subsequent digits, the slope was less steep. To show this change in slope was significant, we analyzed the per-participant slopes between adjacent digits in the identification-rate-by-position curve. As predicted, the first slope (between $1^{\text {st }}-2^{\text {nd }}$ digits) was significantly steeper than the second slope (between $2^{\text {nd }}-3^{\text {rd }}$ digits), at $50 \mathrm{~ms}$ (4-digit numbers: paired $\mathrm{t}(23)=2.73$, one-tailed $p=.006 ; 5$-digit numbers: paired $\mathrm{t}(23)=3.76$, one-tailed $p<.001$ ) and at $100 \mathrm{~ms}$ (4-digit numbers: paired $\mathrm{t}(23)=4.21$, one-tailed $p<.001 ; 5$-digit numbers: paired $\mathrm{t}(23)=2.91$, one-tailed $p=.003)$. At $200 \mathrm{~ms}$, the slope stabilized, suggesting that by $200 \mathrm{~ms}$ the second digit was already processed well enough to overcome the outer-digit advantage. This change of slope was unique to the first digit; the next pair of adjacent slopes $\left(2^{\text {nd }}-3^{\text {rd }}\right.$ versus $\left.3^{\text {rd }}-4^{\text {th }}\right)$ did not differ from each other (5-digit numbers: for all exposure durations, paired $t(23) \leq 1.29$, one-tailed $p>.10$; we did not test this in 4-digit numbers to avoid involving the unit digit).

To summarize, the results supported the two hypotheses presented in the Introduction: the digits in a digit string are identified serially from left to right, but there is also an advantage for the outer digits.

The degree of the left-to-right gradient in the digit identification (reflecting the degree of seriality under the serial processing model) can be estimated as the slope of the identification-byposition curve (excluding the outer digits, as they are prone to the outer-digit advantage). To determine whether longer numbers induced a stronger gradient (i.e., in more serial processing), we computed the slope for each participant separately for 4- and 5-digit numbers, by regressing the digit identification rate against the digit position. The slopes of this regression for 5-digit numbers (mean $=8.2 \%, \mathrm{SD}=5.2 \%$ ) were marginally steeper than for 4-digit numbers (mean $=6.7 \%$, $\mathrm{SD}=7.1 \%$, paired $\mathrm{t}(23)=1.51$, one-tailed $p=.07$ ), suggesting longer numbers may be processed slightly more serially than shorter numbers. This finding agrees with previous studies finding serial processing in long but not short numbers (e.g., Meyerhoff et al., 2012). Here, we saw a length effect even within the numbers previous studies considered "long" (4+ digits).

In principle, there is some uncertainty about coding errors as position errors or identification errors, because random substitutions may occasionally just happen to be identical with another digit in the target number (e.g., in the number 42 , substituting $4 \rightarrow 2$ and forgetting the 2 would result in twenty something and thus be coded as migration). However, clearly the position errors in our data should not be interpreted this way: the number of position errors out of all errors (identification and position) was higher than expected under the assumption of random substitutions (binomial $p<.001$ ). Furthermore, as the next section shows, the patterns of position errors and identification errors were similar. To verify unambiguously that the findings were not an artefact of our coding scheme, we examined accuracy (whether the target digit was produced correctly and in the correct position), thus avoiding any potential confusion between identification and position errors. The accuracy data followed the same error-by-position pattern as identification 
and position errors (Fig. 3c), and when using accuracy as the dependent variable, we essentially replicated all the statistical analyses reported above.

\subsubsection{Digit position encoding}

We next examined the encoding of digit positions, still for numbers without 0 and 1 . Fig. $3 b$ shows the rate of digits produced in the correct position out of all identified digits, separately for each target position. The pattern is very similar to the pattern observed for digit identification; position encoding deteriorates for digits farther to the right, with a slight improvement in the unit digit versus the decade digit in 4-digit and 5-digit numbers.

We examined these effects using similar statistical analyses to those described above for digit identification. First, we submitted the data of identified digits to an LLMM (weighting each participant's data as described in Section 0), with position encoding (correct/incorrect) as the dependent variable. The participant was a random factor, and there were 4 within-subject factors: digit position as a numeric factor, outer vs. inner digit, number length (non-numeric), and exposure duration (non-numeric). Supporting the serial-processing hypothesis, the Position factor was significant (odds ratio $=2.13, \chi^{2}=436.7, p<.001$; full results in Table S6). In accord with the outer-digit advantage hypothesis, the Outer/Inner factor was significant as well (odds ratio $=6.90$, $\chi^{2}=484.4, p<.001$ ). Both effects remained significant when we analyzed each number length and exposure duration separately and when we excluded trials with number-length errors (all $p<.001$; 3-digit numbers were not analyzed because they did not show a clear trend in Fig. 3c).

We also examined the outer-digit advantage hypothesis by comparing the position encoding for the decade and unit digits. We used LLMM on the position encoding with the participant as a random factor and 3 within-subject non-numeric factors: digit position (decade or unit), exposure duration (non-numeric), and number length (non-numeric). The Position factor was significant (odds ratio $=0.33, \chi^{2}=96.4, p<.001$; separately for 4- and 5-digit numbers, $\chi^{2}>17.5, p<.001$; full results in Table S8); i.e., as predicted by the outer-digit advantage hypothesis, the digit position encoding was better for the unit than the decade digit.

\subsubsection{The position effect is not a verbal confound}

Both digit identification and digit position showed a monotonous decline in performance in digits farther to the right. In our view, this pattern indicates that the visual analyzer processes the digit from left to right. An alternative interpretation, which we now refute, attributes the effect to memory rather than to the visual analyzer; it suggests that the monotonous decline in performance reflects the fact that our task required saying the numbers aloud. Because digits farther to the right are said later than the digits on the left, the right digits must be retained in memory for a longer period until they are produced, and during this time, their representation in memory may decay. Statistically, this interpretation postulates that the factor affecting performance is not the position 
of the digit in the digit string, but the position of the corresponding number word in the verbal number.

To assess this alternative interpretation, we analyzed numbers that included the digit 0 . In Hebrew, as in English, the digit 0 is not pronounced. In numbers with 0, this creates a discrepancy between the position of the digits 1-9 in the digit string and the position of the corresponding number words in the verbal number. For example, for 305 , " 3 " is the first digit and the first number word, yet " 5 " is the third digit but the second number word. This discrepancy allows us to examine which is the better predictor of digit identification: the digit position or the word position.

We analyzed the inner digits of 4- and 5-digit numbers that included 0 (except when 0 was in the unit position) and did not include 1 . We did not analyze the digit 0 itself. We submitted the digit identification data to an LLMM with the participant as a random factor and 4 within-subject factors: number length, exposure duration, digit position (we used numeric factor, but obtained similar results when it was non-numeric), and number-word position. The Digit Position effect was significant (odds ratio $=1.79, \chi^{2}=37.0, p<.001$; full results in Table S9), but the Word Position effect was not (odds ratio $=1.18, \chi^{2}=1.03, p=.31$ ). Namely, the position of the digit in the digit string predicted its identification rate, with no additional contribution of the number word position. To verify that these analyses were not biased by error trials (e.g., when there is a numberlength error, such as reading 12 as 102, the digit/word position may be different in the target and response), we ran the analysis again, this time excluding items with number-length errors and using accuracy as the dependent variable (correct identification and position). The results were essentially the same (Digit Position effect: odds ratio $=2.02 ; \chi^{2}=31.3, p<.001$; full results in Table S10; the Word Position effect was negative and non-significant). This clearly refuted the alternative interpretation and confirmed our initial conclusion that the deterioration in the processing of digits farther to the right was a visual rather than a verbal effect.

\subsubsection{Interim summary}

The analysis of identification errors and position errors showed a mixture of two effects: the performance deteriorated in digits farther to the right, and there were fewer errors in outer than inner digits.

The decline in performance in positions farther to the right indicates that digits are processed serially, in a left-to-right manner (in the Discussion, we refer to alternative interpretations of this finding). The better performance in the unit digit relative to the decade digit can be explained as an effect of crowding, whereby the unit digit and the leftmost digit are less crowded than the inner digits. Crowding, also known as lateral masking, assumes that the visual information from one digit may disturb the identification of nearby digits (Estes et al., 1976; Gomez et al., 2008; Hammond \& Green, 1982; Townsend et al., 1971; Tydgat \& Grainger, 2009; Wolford, 1975; Wolford \& Hollingsworth, 1974b). In the Discussion, we consider an alternative interpretation of 
the outer-digit advantage effect, but as the next section shows, additional findings supported the crowding interpretation.

\subsection{Additional characteristics of errors in 2-9}

\subsubsection{Digits tend to migrate to adjacent locations}

The analyses above centered on the rate of digit position errors (migrations). In our next step, we examined the destinations of these digit migrations. Fig. 4 shows the frequency of each destination position for the digits 2-9 that migrated. The migrations were clearly not to random positions. To show this, we computed the number of migration errors from each source position to each destination position (using the Fig. 4 matrices, but with raw numbers rather than percentages). We compared each of the two matrices (4-digit numbers, 5-digit numbers) to an $\mathrm{H}_{0}$ matrix that assumed migration to random destinations. The diagonal, reflecting correct responses, was excluded. We created the $\mathrm{H}_{0}$ matrix from the observed matrix by keeping the total number of migrations in each column (each source position) with uniform distribution across rows (destination positions), excluding the diagonal. The observed migration matrices were significantly different from the $\mathrm{H}_{0}$ matrices (5-digit numbers: $\chi^{2}(15)=377.9, p<.001$; 4-digit numbers: $\left.\chi^{2}(8)=161.0, p<.001\right)$, confirming the migrations were not to random positions.
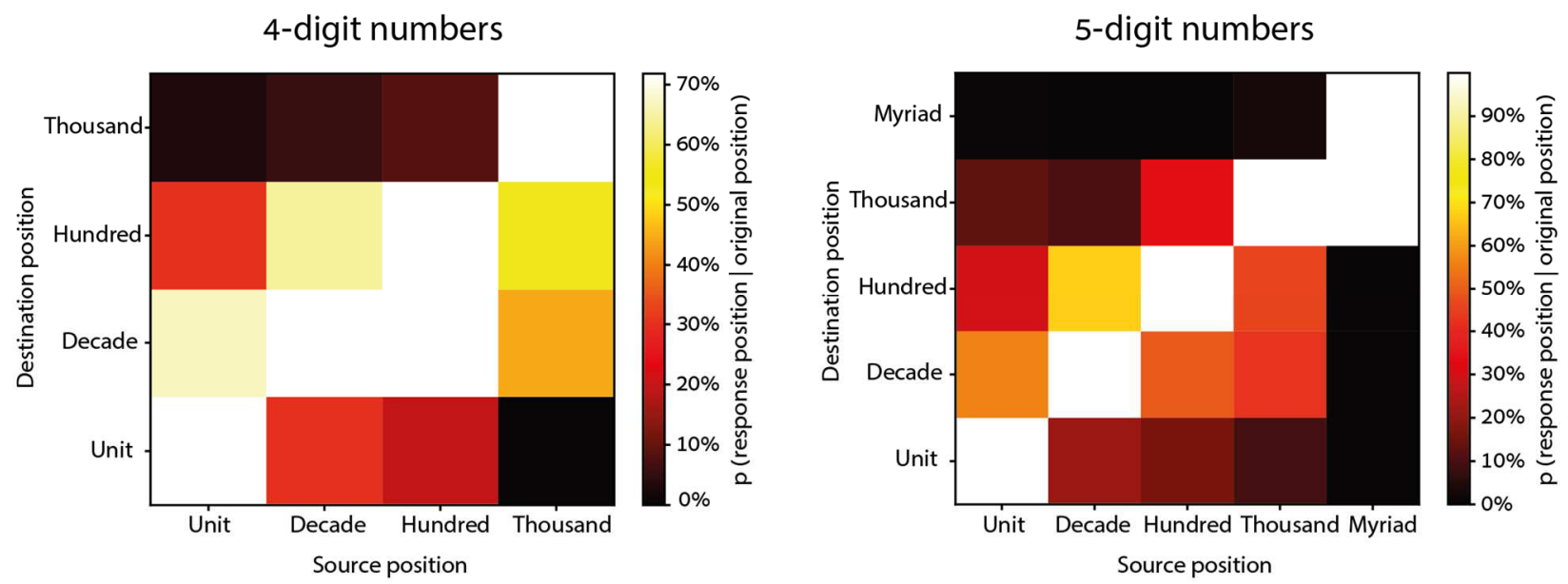

Fig. 4. Source and destination positions of digits identified in incorrect positions, across all participants. The colors denote the conditional probability that, if a digit in position $\mathrm{X}$ migrated, it was to position $\mathrm{Y}$ (i.e., each column sums to $100 \%$, and the color of the diagonal is meaningless). Migrations to adjacent locations were more frequent than to farther locations.

A closer inspection of Fig. 4 suggests that in line with previous reports (Friedmann et al., 2010), digits tended to migrate to adjacent positions: $83 \%$ of the migrations in 4-digit numbers and $72 \%$ of the migrations in 5-digit numbers were to adjacent positions. The rate of adjacent migrations was significantly higher than the chance level predicted by assuming migration to random positions $(5$-digit numbers: chance $=50 \%, \mathrm{t}(23)=4.85$, one-tailed $p<.001 ;$ 4-digit 
numbers: chance $=66.7 \%, \mathrm{t}(23)=8.66$, one-tailed $p<.001)$. These results were replicated, with stronger significance levels, when we included numbers with 0 and/or 1 .

\subsubsection{Greater confusion between visually-similar digits}

We next examined whether the errors were affected by the visual properties of the digits. Did participants tend to substitute or transpose a digit with a visually similar digit? We first determined whether when the participants substituted a digit, they responded with random digits (Fig. 5). If substitutions were random, the distribution of responses should have been uniform for each target digit (i.e., within each column in Fig. 5), but this was not the case. To show this, we analyzed the target-response distribution for all erroneous digits, excluding situations in which either the target or the response was 0 or 1 (i.e., excluding the two bottom rows in the Fig. 5 matrix, the two left columns, and the diagonal). We compared the remaining $8 \times 7$ matrix to an $\mathrm{H}_{0}$ matrix reflecting the assumption of random substitutions. The $\mathrm{H}_{0}$ matrix was generated by keeping the total occurrences of each target (column) but ensuring uniform distribution across response digits (rows). The observed matrix was significantly different from the $\mathrm{H}_{0}$ matrix $\left(\chi^{2}(48)=320.0, p<.001\right)$, confirming the substitutions were not random.

To specifically examine whether participants tended to substitute a digit with a visuallysimilar one, we examined the 10x10 substitution matrix, excluding the diagonal (correct responses). To control for the uneven distribution of target digits, we normalized the matrix (as in Fig. 5) to get a fixed sum for each column. To control for possible bias towards particular response digits, we normalized the matrix to get a fixed sum for each row. We compared this transformed substitution matrix with a 10x10 matrix of the subjective visual similarity between pairs of digits (visual similarity data from Godwin et al., 2014). The transformed substitution matrix and the visual similarity matrix were correlated, even if the correlation was not high (Pearson $r=0.20^{1}$, also when excluding the digits 0 and $1(\mathrm{r}=0.25)$. Namely, when a substitution error occurred, the response was likely to be a digit that was visually similar to the target digit.

We also examined whether the response digit tended to be numerically similar to the target digit (a distance effect). It was not. To show this, we regressed the digit substitution matrix of the digits 2-9, excluding the diagonal, against two predictors: the log of the absolute value of the targetresponse digit numerical distance; and the visual similarity between the target and response digits. The Distance predictor had almost no effect, and its small effect was opposite to the predicted direction (regression coefficient $\beta=.015$ ), but visual similarity still had an effect $(\beta=.25$ ).

\footnotetext{
${ }^{1}$ Here and in subsequent statistical analyses of the error-distribution matrix, we do not report $p$ values because the matrix cells are not statistically independent.
} 


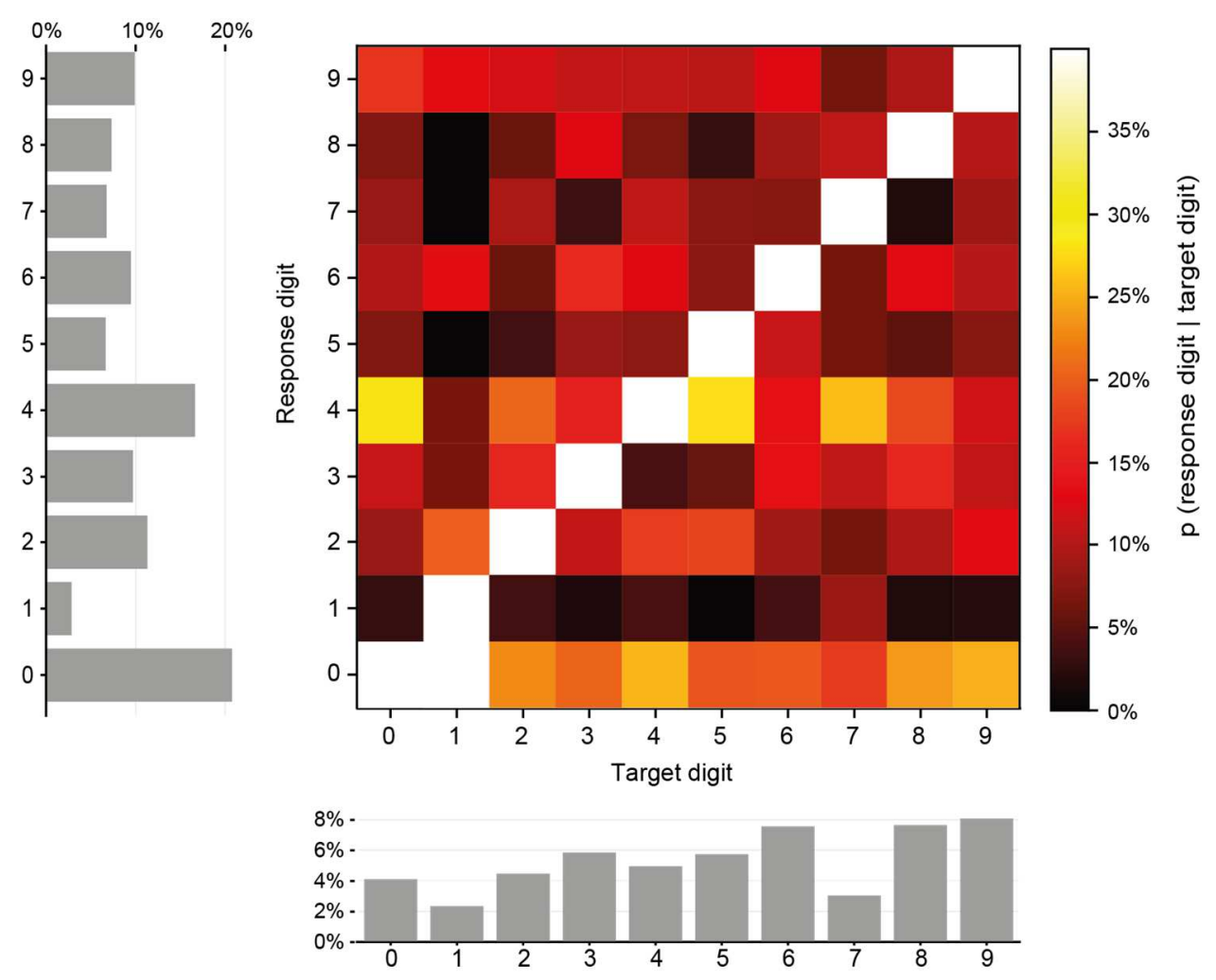

Fig. 5. Distribution of erroneous response digits, across all participants, in cases of substitution errors (i.e., excluding correctly identified and unidentified digits). The colors indicate the distribution of responses given a particular target digit (conditional probability); i.e., each column sums to $100 \%$, and the diagonal color is meaningless. Below the matrix: the percentage of identification errors for each target digit. To the left: the histogram of response digits (percentage out of all substitution errors).

Although visual similarity affected the substitution errors, it did not show a significant effect on position errors: visually-similar digits were not necessarily more likely to transpose with each other. We examined all the pairs of identified digits in each number, excluding pairs in which one of the digits was 0 or 1 and excluding 3-digit numbers. The point biserial correlation between visual similarity and transposition (i.e., whether the digit was transposed or not) digits was very low (4-digit numbers: $r=0.003 ;$-digit numbers: $r=.03$ ).

\subsubsection{Sensitivity to distribution of target digits}

Within the substitution errors, the distribution of response digits (Fig. 5, left bars) correlated with the distribution of target digits in the experiment: excluding the digits 0 and 1 , the two histograms correlated significantly $(r=.68)$. This suggests participants were sensitive to the distribution of digits in the experiment, echoing previous findings (Dotan \& Dehaene, 2020a).

We refuted an alternative interpretation attributing this correlation to the perseveration of digits from the target of the recent trial. The rate of digits that could be explained as recent-target perseverations $(63.5 \%)$ was not higher than the chance level $(50.6 \%$, one-tailed $p=.17)$. We 
computed the chance level and $p$ value using a bootstrap method. For each subject, we shuffled the pairing of a response digit with a previous target 10,000 times while maintaining the previous target number length, and we computed the probability that such random shuffling would produce at least as many as many perseverations as were observed. Perseverations were also not more frequent than chance when we excluded the digits for which the response was 0 or $1(p=.21)$, or when we examined perseverations from the previous trial's response rather than the target $(p=.50)$.

\subsubsection{Better identification of small digits}

(a)
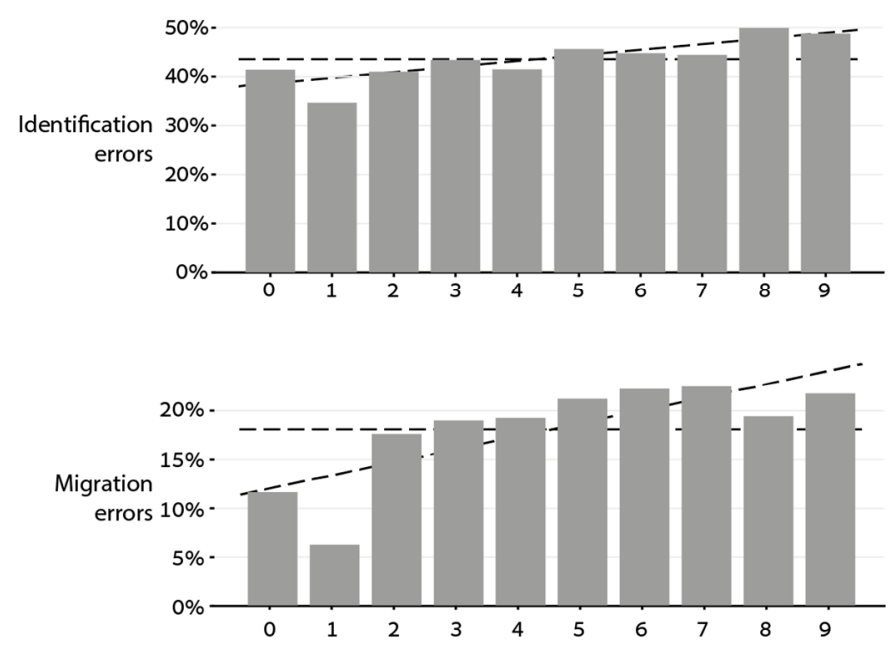

(b)
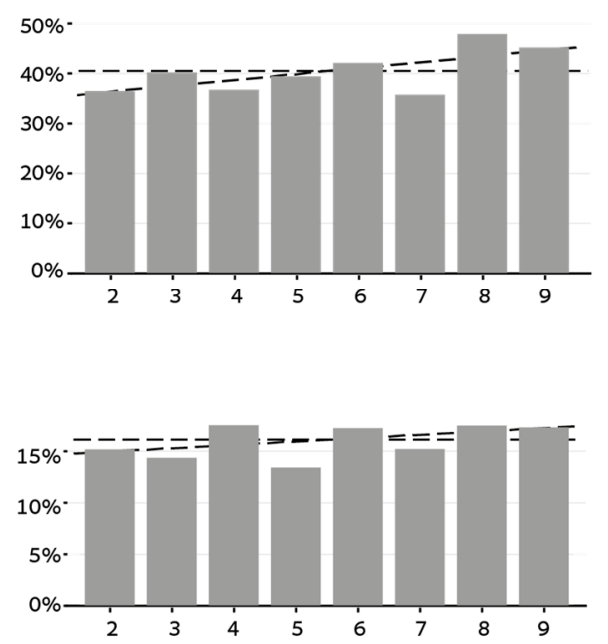

Fig. 6. Rate of identification errors and position errors in each target digit, for (a) all items, excluding the leftmost digit in each number (which was never 0 and could create a bias), and (b) items that did not include 0 and 1. The digits 0 and 1 had fewer position errors than 2-9; the digit 1 also had fewer identification errors. Horizontal dashed lines show the mean of all bars. Tilted dashed lines show a linear fit based on the digits 2-9, indicating better performance in smaller numbers.

We examined whether some of the digits 2-9 were more prone to errors than others ( 0 and 1 are not analyzed here because they are discussed in detail in the next section). Fig. 6 suggests the likelihood of errors was affected by the digit magnitude, whereby larger digits were more errorprone. To examine this effect statistically, we submitted the digit identification data of numbers without 0 and 1 (Fig. 6b) to an LLMM with the participant as a random factor and 5 within-subject factors. The critical factor was the target digit as a numeric factor (i.e., the digit magnitude). Additional factors were the digit position (numeric factor), outer vs inner digit, number length, and exposure duration. The Magnitude effect was small and marginally significant (odds ratio $=0.98$, $\chi^{2}=3.5, p=.06$; full results in Table S11); i.e., smaller digits were identified slightly better. 


\subsubsection{Interim summary}

The strong tendency of digits to migrate to adjacent locations supports the notion that visual information leaks from each digit to the processes identifying the adjacent digits (Davis, 2010; Gomez et al., 2008; Ktori et al., 2014; Tydgat \& Grainger, 2009). Such leaks could cause a digit to be incorrectly identified as its neighbor, and we would code this as adjacent migration. Errors were also affected by the digit magnitude - smaller digits had fewer identification and position errors. A simple explanation is that smaller digits are processed better because they are more frequent (Dehaene \& Mehler, 1992).

\subsection{Processing the digits 0 and 1}

We now turn to the performance in the digits 0 and 1, comparing this to the other digits. These two digits have special roles in the number system, and the main question in this part is whether the visual analysis process treats them differently. Is the processing of 0 and 1 superior to that of the other digits?

\subsubsection{Identifying 0 and 1}

We started by analyzing digit identification. If a dedicated process helps identify the identities of 0 and 1 , there should be fewer identification errors for 0 and 1 than other digits. Numerically, the identification error rate for 0 and 1 was lower than the average of the other digits (Fig. 6a). However, as we shall now see, this difference was weak or non-significant.

We first examined 0 versus 2-9. The digit identification data for 4- and 5-digit numbers was submitted to an LLMM (Section 0) with the participant as a random factor and 7 within-subject factors. The critical factor was Zero - a binary factor indicating whether the digit in question was 0 or not. Additional factors were digit position (numeric factor); inner vs outer digit; exposure duration (non-numeric factor); number length (non-numeric factor); the digit as a numeric factor to control for the possibility of fewer identification errors in smaller numbers (Section 3.2.4); and the average visual similarity between the target digit and all other digits (Table S2) to control for the possibility that 0 is visually more distinctive than other digits. We excluded the leftmost digit, which is easy to identify but was never 0 , and the digit 1 , to avoid confounding the research questions about 0 and 1 . The Zero effect was negative and not significant (odds ratio $=0.89$, $\chi^{2}=2.0, p=.16$; full results in Table S12); i.e., 0 was not better identified than the digits 2-9.

We used the same LLMM to compare the identification of 1 to the identification of the digits 2-9. We excluded the 3-digit numbers, the digit 0 , and the leftmost digit. The Zero factor was replaced by a One factor, and its effect was significant (odds ratio $=1.69, \chi^{2}=27.2, p<.001$; full results in Table S13) - i.e., the digit 1 was identified better than the digits 2-9. Note that although the role of 0 in the number system is more central than that of 1 , the digit 1 was identified better than 0 (for the same type of LLMM with only 0 and 1 included, without the magnitude and visual similarity factors: odds ratio $=1.75, \chi^{2}=21.6, p<.001$; full results in Table S14), suggesting 
additional factors were at play - perhaps the visual shape of 1 , which is more distinctive than 0 (Table S2).

Using a similar analysis, we examined whether any of the digits 2-9 was identified better than the other digits. The digits 0 and 1 were excluded from this analysis. Except the digit $7\left(\chi^{2}=43.57\right.$, $p<.001)$, no other digit was better identified than the others $\left(\chi^{2}<4.49, p>.23\right.$ after BonferroniHolm correction for 8 comparisons). The better identification of the digit 7 may be explained by its visual distinctiveness (see Table S2).

\subsubsection{Encoding the positions of 0 and 1}

The next question was whether the digits 0 and 1 are treated in a special and superior manner in position coding. This was clearly the case (Fig. 6a): the position detection of 0 and 1 was dramatically better than 2-9. To show this difference was significant, we used the same type of analysis as above for digit identification. First, we examined 0 versus 2-9. The position-detection data of the identified digits in 4- and 5-digit numbers were submitted to an LLMM (Section 0) with the participant as a random factor and 6 within-subject factors. The critical factor was Zero a binary factor indicating whether the digit in question was 0 or not. Additional factors were digit position (a numeric factor), inner vs outer digit, exposure duration, number length, and the digit as a numeric factor (to control for the magnitude effect). We excluded the leftmost digit in each number and the digit 1, and we weighted the data points as described in Section 0. The Zero effect was significant (odds ratio $=1.66, \chi^{2}=20.5, p<.001$; full results in Table S15); i.e., there were fewer position errors for 0 than 2-9.

A similar analysis showed the position encoding of 1 was superior to the digits 2-9. We analyzed the 4 - and 5-digit numbers excluding the digit 0 , the leftmost digit, and unidentified digits. The Zero factor was replaced by a One factor, and its effect was significant (odds ratio = 5.07, $\chi^{2}=91.1, p<.001$; full results in Table S16). The position of 1 was also encoded better than the position of 0 (a similar analysis that included only the digits 0 and 1; Zero effect: odds ratio = 2.92, $\chi^{2}=28.7, p<.001$; full results in Table S17).

Among the digits 2-9, no digit showed position encoding superior to the others (same method as above, applied to each of the digits 2-9: $\chi^{2}<5.69, p>.13$, Bonferroni-Holm corrected for 8 comparisons; 0 and 1 excluded from analysis).

\subsubsection{How 0 and 1 affect other digits}

The analyses above showed that the position of 0 and 1 was encoded much better than the position of 2-9, suggesting that 0 and 1 are handled by dedicated processes. A possible prediction is that the existence of 0 or 1 in a number would also facilitate the processing of the other digits. For example, if there are two mechanisms, one for processing 0 and 1 and another for processing $2-9$, the latter mechanism may have an easier job when the number includes 0 or 1 , and, thus, it may operate better. 
Fig. 7 suggests this was indeed the case; the identification of 2-9 was better when the number included 0 or 1 . To show the facilitating effect of 0 was statistically significant, we submitted the digit identification data of the digits 2-9 in the 4-digit and 5-digit numbers to an LLMM (Section 0 ) with the participant as a random factor and 6 within-subject factors. The critical factor was Zero - a binary factor indicating whether the number included 0 or not. Additional factors were digit position (numeric factor), outer vs inner digit, exposure duration (non-numeric factor), number length (non-numeric factor), and the digit as a numeric factor (to control for the magnitude effect). The Zero effect was significant (odds ratio $=1.28, \chi^{2}=43.9, p<.001$; full results in Table S18), confirming that 0 in the number facilitated the processing of the other digits. A similar analysis for the digit 1 showed its effect was significant as well (odds ratio $=1.31, \chi^{2}=23.9, p<.001$; full results in Table S19). No other digit showed a similar effect $\left(\chi^{2}<3.01\right.$, Bonferroni-Holm corrected $p>$.66). The facilitating effects of 0 and 1 were not significantly different from each other (same LLMM, including only numbers with 0 or 1 but not both, and without the digit numeric factor: digit effect odds ratio $=0.99, \chi^{2}=0.01, p=.91$ ). The right column in Fig. 7 shows how much the existence of each digit in the number contributed to the identification of the digits 2-9, according to the LLMM described here. We can clearly see the facilitating effect of 0 and 1.
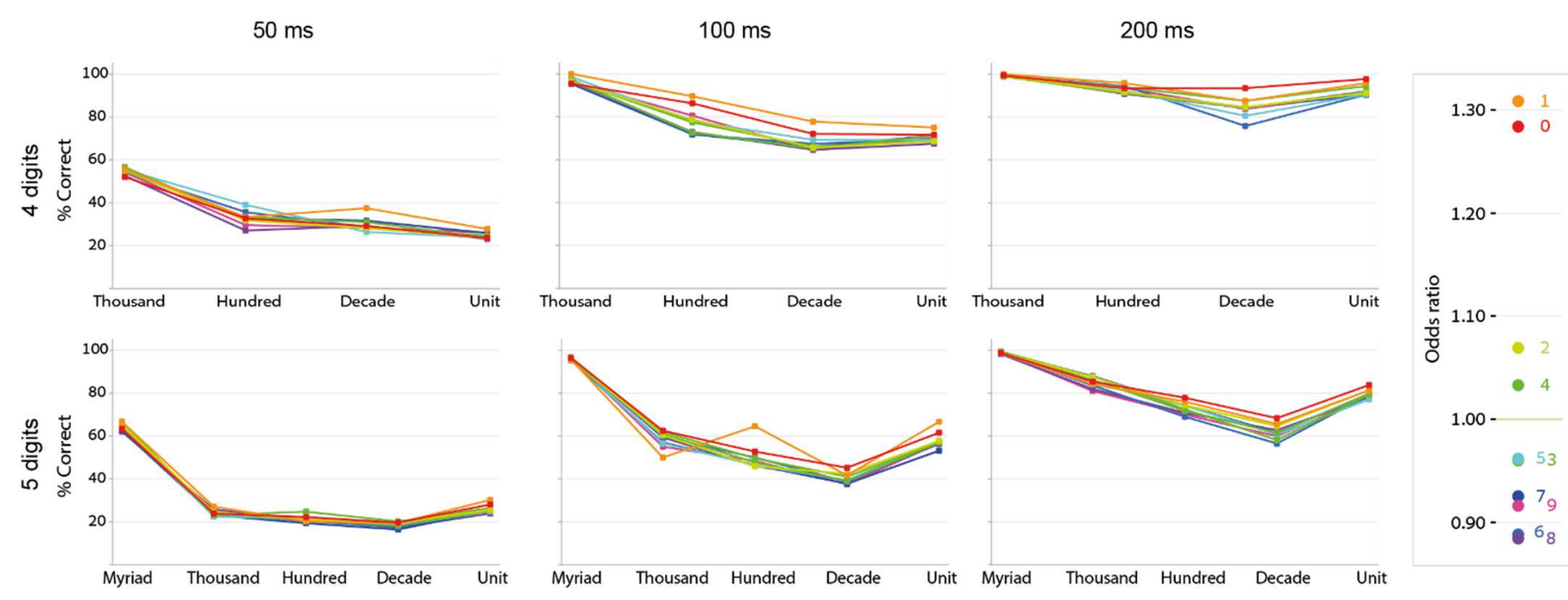

Fig. 7. Identification rate of the digits 2-9 in numbers with a particular target digit. The box on the right shows the identification rate gained when each digit was in the number, presented as the odds-ratio of that digit's factor in the logistic linear mixed model described in the text. We can see that the presence of 0 or 1 in the number improved the identification of the other digits.

How exactly do 0 and 1 contribute to the identification of the other digits in the number? One possibility explains this effect in terms of the interference between adjacent digits, as proposed in Section 3.2.5. If the visual analysis tends to confuse adjacent digits due to leakage of visual information from a digit to its neighbors, there may be an inhibition mechanism that aims to 
prevent this leakage: once the visual analyzer identifies a digit $\mathrm{X}$ in a certain position, this protective mechanism would inhibit the digit $\mathrm{X}$ in adjacent positions. Because 0 and 1 are particularly important, this protective adjacent-digit inhibition is particularly strong for these two digits.

An interesting prediction of this hypothesis is that the facilitating effect of 0 and 1 on the other stimulus digits should not be equal across all positions. Rather, 0 and 1 should especially facilitate the identification of adjacent digits, perhaps only the adjacent digits. We examined this prediction separately for 0 and for 1 . For 0 , we considered the digit identification data of the digits 2-9 in 4and 5-digit numbers that included 0 . We excluded numbers with 1 , to avoid confounding the effect of 0 with that of 1 . The digit identification data were submitted to an LLMM with the participant as a random factor and 6 within-subject factors. The critical factor was Adjacency, a binary factor indicating whether the digit in question was adjacent to 0 or not. Additional factors were digit position (numeric factor), outer vs inner digit, exposure duration, number length, and the digit as a numeric factor. The Adjacency effect was significant (odds ratio $=1.2, \chi^{2}=7.8, p=.005$; full results in Table S20), confirming that, as predicted, 0's facilitating effect was the strongest for the digits next to it. A similar analysis examined the effect of 1 on its neighbors. We used the same type of LLMM, now with the numbers containing 1 but not 0 . The Adjacency effect was marginally significant (odds ratio $=1.26, \chi^{2}=3.2, p=.08$; full results in Table S21). Namely, like 0, the facilitating effect of the digit 1 on other digits was driven by its effect on adjacent digits, but to a lesser extent than we saw for 0 . Importantly, the Adjacency effect was unique to 0 and 1: in a series of 8 similar analyses for the other digits (excluding the numbers with 0 or 1), the digits 2-9 did not show any adjacency effect $\left(\chi^{2} \leq 1.64\right.$, Bonferroni-Holm corrected $\left.p=1.0\right)$.

Do 0 and 1 facilitate only the processing of adjacent digits, or do they affect farther digits too? To examine this question, we compared the digit identification of numbers with 0 to numbers without 0 (and the same for 1). Crucially, we only considered digits not adjacent to 0 (or 1). First, to examine the effect of 0 , we ran an LLMM on the digit identification data of the digits 2-9 in 4and 5-digit numbers that included 0 but not 1 , excluding the digits adjacent to 0 . The participant was a random factor, and there were 6 within-subject factors. The critical factor was Zero - a binary factor indicating whether the number contained 0 or not. Additional factors were digit position (numeric factor), outer/inner digit, exposure duration, number length, and the digit as a numeric factor. The Zero effect was significant (odds ratio $=1.14, \chi^{2}=7.64, p=.006$; full results in Table S22). We ran a similar LLMM to examine the effect of 1 on non-adjacent digits, now including only numbers with 1 and without 0 and excluding the digits adjacent to 1 . The One effect was significant (odds ratio $=1.18, \chi^{2}=4.79, p=.03$; full results in Table S23). Namely, both 0 and 1 improved the identification of non-adjacent digits, although the effect was smaller than for adjacent digits. 
To summarize, the digits 2-9 were identified better when the number included 0 or 1 than when it did not. This facilitating effect of 0 and 1 was the strongest for the digits next to them, but was observed for non-adjacent digits as well.

The finding that 0 facilitates the processing of adjacent digits has one particularly important consequence: this effect cannot be attributed to the verbal production processes, because 0 is not produced. In other words, the facilitating effect of 0 on adjacent digits clearly occurs in the visual analyzer, not in verbal production processes.

\section{Discussion}

\subsection{Summary of findings}

This study examined in detail the processes we use to visually analyze digit strings when we read multi-digit numbers. Participants read aloud 3-, 4-, and 5-digit numbers presented in short exposure durations. The main findings were as follows.

1. Digit position affected performance. The rate of digit identification errors (failing to identify a digit) and digit position errors (saying a digit in the incorrect decimal position) was not uniform across decimal positions. It was affected by a mixture of two factors:

Distance from leftmost digit. The performance decreased continuously with the digit's serial position in the number: the farther a digit was from the number's left side, the lower the performance (except for the unit digit). This effect of serial position was observed in numbers of all lengths but was slightly stronger for longer numbers. Crucially, the performance was best predicted by the position of the digit in the digit string, not by the position of the corresponding number-word in the sequence of words. Namely, although the reading-aloud task includes both the visual input of digit strings and the verbal production of number words, the serial position effect should be interpreted as a property of the visual analysis of the digit string, not of the verbal number production. The serial position effect was observed in numbers that included only the digits 2-9; i.e., the particularities of 0 and 1 cannot account for the serial position effect.

Outer-digit advantage. The performance in the two outer digits - the rightmost (units) and the leftmost - was better than the performance in the inner digits. On the right side, the outer-digit advantage was large enough to outweigh the distance-from-left-digit effect described above: although the unit digit is farther to the right than the decade digit, in most conditions (particularly in the longer numbers, with 4 and 5 digits), the performance in the units was better than in the decade digits. Indeed, the outer-digit advantage increased with longer numbers, even if not by much. For the leftmost digit, the outer-digit advantage and the distance-from-left effect operated in the same direction, and, correspondingly, the performance in the leftmost digit was much better than in the second digit. 
2. Adjacent migrations were more frequent than nonadjacent migrations. When the participants made position errors, the target digits most often migrated to an adjacent position (either leftwards or rightwards), less often to farther positions. This effect parallels the adjacency effect in word reading (Ktori et al., 2014; Perea et al., 2008). The migration-by-position pattern (Fig. 4) suggests this distance effect is continuous: adjacent migrations are the most frequent, twostep migrations seem to be more frequent than three-step migrations, and so on. However, this possibility was hard to test, as migrations were subject to the outer-digit advantage effect, and our experiment only included numbers up to 5 digits.

3. Digit position was identified better for 0 and 1 than for 2-9. Digit position errors were less frequent for the target digits 0 and 1 than for other digits. For the digit identification errors, only the digit 1 showed similar superiority, but the effect was still stronger for position than substitution errors. Although there was a general tendency for better performance in smaller numbers (see below), this effect was not sufficient to account for the superior performance in 0 and 1.

4. Better performance in digits near 0 or 1. There were fewer identification and position errors in the digits 2-9 when the number included 0 and 1, especially when the target digit was adjacent to 0 or 1 . Although the facilitating effect of 0 and 1 on their neighbors extended to nonadjacent digits, it was stronger for adjacent ones. The facilitating effect of 0 on other digits in the number can be safely attributed to the visual analyzer (not to verbal production), because 0 is not produced verbally.

5. Digits tend to be substituted by visually-similar digits. When a substitution error occurred, the target digit was more likely to be substituted by a visually-similar digit than by a visually dissimilar digit. This replicates previous studies showing the effect of the visual similarity between characters when reading numbers (but not words; Kinoshita \& Norris, 2009; Kinoshita et al., 2014).

6. Better performance in smaller digits. There were fewer digit identification errors and digit position errors in digits with smaller values. Because smaller numbers are more frequent (Dehaene \& Mehler, 1992), this finding may reflect an effect of frequency. Alternatively, it may reflect the superior processing of smaller magnitudes (Dehaene, 2007). However, because our task did not require attending to magnitude (and certainly not to the magnitudes of individual digits), this interpretation seems less likely.

Importantly, as explained above, our data indicate that the main findings - the serial-like processing pattern and the special status of the digit 0 - originate in the visual analyzer and not in verbal production processes. Future studies may directly corroborate and extend this conclusion by examining whether similar patterns exist also in tasks that require visual analysis and do not require any verbal production. 


\subsection{Properties of the visual analyzer}

The findings lead to the following conclusions.

\subsubsection{Digits are processed serially, from left to right}

The encoding of digit identity and digit position was better for digits on the left and decreased continuously for digits farther to the right. Our best explanation of this pattern is that the digits in a multi-digit string are processed from left to right, with temporal overlap (i.e., the processing of the second digit starts before the first digit is fully processed, and so on). In a limited-exposure paradigm, as in our study, such seriality means there is more time to process the digits on the left; consequently, there are fewer errors in digits on the left. Indeed, errors-by-position curves similar to ours were observed in studies specifically designed to encourage serial processing (e.g., Wolford \& Hollingsworth, 1974a). Note that we do not claim all processing stages within the visual analyzer are serial: it is possible that only some visual analysis sub-processes impose a seriality bottleneck, whereas others operate in parallel (Davis, 2010; Tydgat \& Grainger, 2009; Whitney, 2001; Wolford, 1975).

This pattern - the monotonous decrease in the performance-by-position curve - was observed for all decimal positions except the unit digit: for numbers with 4 digits or more, the performance in the unit digit was better than in the decade digit. This deviation from monotony does not refute the assumption of seriality, because it can be explained by other factors - e.g., visual crowding. We discuss this below.

With unlimited exposure, adults make very few errors in reading aloud multi-digit numbers, such as the numbers we used here (e.g., errors in less than $3 \%$ of the numbers in Dotan \& Friedmann, 2018). Our participants made errors even for the longest exposure duration (200 ms); i.e., $200 \mathrm{~ms}$ was not sufficient for optimal processing. Merely the existence of errors cannot indicate which specific process requires more than $200 \mathrm{~ms}$ (e.g., whether it is the visual analyzer or the verbal production). However, our finding that digits tended to be substituted with visuallysimilar digits suggests that $200 \mathrm{~ms}$ was not enough time even for the stage of digit identification. The idea that the accumulation of visual evidence is an imperfect process requiring sufficient time, even when the stimulus is a clearly visible symbol, has been demonstrated in other paradigms as well (Dotan \& Dehaene, 2016; Drugowitsch et al., 2016).

Previous studies examining Arabic number reading have had mixed results for serial versus parallel processing of the digits. Studies focusing on numbers with 4 digits and more have found evidence of serial processing of the digits, similar to our findings here (Friedmann et al., 2010; Meyerhoff et al., 2012). Studies using 2-digit and 3-digit numbers, in a variety of paradigms, have generally concluded the digits are processed in parallel (Dehaene et al., 1990; Dotan \& Dehaene, 2020; Moeller et al., 2009; Nuerk \& Willmes, 2005; with exceptions in some specific cases for 3digit numbers, Bahnmueller et al., 2016). Thus, it seems the digits are increasingly processed 
serially for longer numbers. Indeed, our results too suggest longer numbers induce more seriality than shorter numbers: in longer numbers, we observed a slightly larger slope of the identificationby-position curve. Still, serial processing has been observed even in two-digit numbers for specific populations: a patient with a focal brain lesion (Dotan et al., 2014) and fourth grade children (Dotan \& Dehaene, 2016). This suggests the ability to process digits in parallel can be attributed to a cognitive mechanism that develops with age or experience and can be selectively impaired following a brain injury.

Serial processing is not the only possible explanation of the left-to-right error gradient. An alternative interpretation could attribute this effect to a spatial bias - e.g., readers may allocate more attention to the left side of the digit string. Furthermore, our participants performed the experiment in Hebrew, in which numbers are read like English from left to right, but words are read from right to left. We might well ask whether the serial-like pattern we observed resulted from this particularity of Hebrew or exists in left-to-right languages too. Although our study cannot answer this question directly, our results agree with the finding of a serial reading pattern in leftto-right readers (Meyerhoff et al., 2012). Future studies may directly compare the serial-processing interpretation versus the attentional-bias interpretation, including the putative effect of word reading direction.

\subsubsection{Interference between adjacent digits}

Our findings support the idea that adjacent digits interfere with each other. This would explain the outer-digit advantage. Simply stated, the processing of each inner digit is disrupted by the two digits next to it, but the processing of an outer digit is disrupted only by one digit, making it easier to encode its identity and position. The idea of adjacent-character interference (often referred to as crowding or lateral interference) is quite common in the literature on word reading and dates back to the 1970 s - including the specific claim that lateral interference underlies the outer-letter advantage effect (Bouma, 1973; Estes et al., 1976; Gomez et al., 2008; Hammond \& Green, 1982; Rumelhart \& McClelland, 1982; Townsend et al., 1971; Tydgat \& Grainger, 2009; Wolford, 1975; some describe this interference as uncertainty about the character's position, e.g., Davis, 2010). Similarly, the idea that lateral interference exists for number reading is not new (Chanceaux \& Grainger, 2012; Tydgat \& Grainger, 2009). Here we extend this idea by showing that adjacent digits not only perturb each other, but also tend to be confused with each other (adjacent migrations).

Interference between adjacent digits presumably arises because the visual information of each digit leaks to the nearby positions (Davis, 2010; Gomez et al., 2008; Ktori et al., 2014; Tydgat \& Grainger, 2009). Such visual leaks may underlie at least some cases of position error and can explain why adjacent position errors were so common in our data. The likelihood of such errors may be even higher when the digit and its neighbor are visually similar - in agreement with our finding that migration errors are more common when digits are visually similar. 
If this kind of visual leakage is abnormally large, it may lead to position errors when reading, causing digit position dysnumeria in number reading (Dotan \& Friedmann, 2018; Friedmann et al., 2010). In an experiment substantiating this suggestion, EY, a woman with digit position dysnumeria (DPD) whose performance we described in detail elsewhere (Friedmann et al., 2010), migrated digits also in the vertical direction, e.g., when asked to locate a digit in a matrix of digits (Dotan and Friedmann, unpublished data). In her case, visual information may have leaked vertically. Note that not all cognitive architectures can easily explain such vertical leakage. For example, if a character's visual receptive field overlaps with that of an adjacent character (Tydgat \& Grainger, 2009), leakage may occur in the vertical direction too; but if the reason for the visual leakage is uncertainty in encoding the character's serial position in the character string (Davis, 2010; Whitney, 2001), vertical leakage is harder to explain. Future studies may perhaps use vertical character migrations to differentiate the multiple reasons for disorders impairing the digit position encoding.

Our data cannot be fully explained by attributing the outer-digit advantage to the order in which the characters are processed (Friedmann \& Gvion, 2001). Such an alternative interpretation emphasizes that when exposure times are limited, a digit that is processed earlier will have more processing time; thus, the performance in this digit will be better (this is essentially the argument we made for seriality in Section 4.2.1). This alternative interpretation views the outer-digit advantage as indicating each outer digit is processed before the digit next to it. However, this alternative interpretation does not fully explain our data, in particular the tendency for adjacent digit migrations and the finding that visually-similar digits are more likely to be transposed. The alternative interpretation also cannot explain specific patterns of errors made by individuals with digit position dysnumeria versus letter position dyslexia (a reading disorder that impairs the encoding of letter positions within a word). Individuals with letter position dyslexia almost exclusively transpose the inner letters, rarely the outer letters, presumably because the earlier processing of the latter "protects" against migration errors (Friedmann \& Gvion, 2001). If digit strings are similarly read in an outer-to-inner manner, we should observe no outer-digit transpositions in individuals with digit position dysnumeria. In fact, these people show almost the opposite pattern: more position errors in the unit digit than in the inner digits (Friedmann et al., 2010). Thus, the letters of a word may be processed in an outer-to-inner order, but this does not seem to be the case for digit strings.

\subsubsection{The digits 0 and 1 are handled by a dedicated mechanism}

Previous studies have proposed that within the visual analyzer, specific mechanisms process the digits 0 and 1 (Cohen \& Dehaene, 1991; Dotan \& Friedmann, 2018). The presumed reason is the special role of 0 and 1 in the subsequent processing stages - the conversion to verbal representation or to quantity representation. Our findings strengthen and extend the idea that 0 and 1 are handled by dedicated mechanisms. First, 0 and 1 were encoded better than the other digits: 1 
was identified slightly better than 2-9, and the position encoding of both 0 and 1 was dramatically better than for the digits 2-9. Second, the digits 2-9 were identified better when they were next to 0 or 1 than when they were not.

The finding that 0 and 1 facilitate the processing of other digits, especially those adjacent to them, is particularly interesting. It suggests that the process dedicated to 0 and 1 within the visual analyzer may be more sophisticated than merely improving the processing of these two digits. We propose that this process aims to prevent the leakage of visual information from 0 and 1 to nearby positions. Thus, whenever the digit 0 or 1 is identified in a certain position, this mechanism inhibits the identification of this digit in nearby positions. Such inhibition should have two consequences, both of which we saw in our data. First, it should reduce the likelihood of 0 and 1 migrating to other positions. Second, it should help the process that identifies digits near 0 or 1 . This process typically has to deal with visual interference (leakage) from nearby digits and should now have an easier job because the inhibition mechanism described above protects against this visual interference from the neighboring 0 or 1 . Our data cannot indicate whether this nearby-digit inhibition mechanism operates on all digits or only on 0 and 1 . However, even if it operates on all digits, its effect is still stronger for 0 and 1, presumably because of their importance.

Our findings agree with several neuropsychological studies showing that even when reading numbers is impaired, the processing of 0 and 1 may be spared (Cohen \& Dehaene, 1991; Dotan \& Friedmann, 2018; Friedmann et al., 2010; Schubert et al., 2020). The case described by Schubert et al. (2020) is particularly interesting: this patient was completely unable to read even single digits, but could still read 0 and 1 .

\subsection{How does the digit visual analyzer operate?}

To accommodate our conclusions, we propose a model for the visual analysis of digit strings. The model rests on existing models of the visual analysis of words and numbers. It employs the common assumption that parsing a character string involves an array of character detectors with a certain degree of vagueness about character-position encoding. The model does not commit to a particular architecture of detectors. One kind of architecture assumes one detector for each decimal position; it outputs the digit identity, with some overlap in the visual receptive fields of adjacent detectors (Dehaene et al., 2005; Tydgat \& Grainger, 2009). Another architecture assumes 10 position-independent detectors, one for each digit. Each detector outputs whether this digit was identified and in which position, with some uncertainty about the position (Davis, 2010; Whitney, 2001). In what follows, we first describe the model under the assumption of one detector per position (Fig. 8) - not because we prefer this specific architecture, but because it is simpler to illustrate. Then we show how the model can be easily adapted to the second architecture.

The input to the digit detectors arrives from an earlier stage that has identified visual features typical to letters and digits (see McCloskey \& Schubert, 2014 for a detailed cognitive architecture 
of the transition from the visual-features level to the digit-identification level). Visually similar digits (i.e., digits sharing visual features) would tend to be confused with each other, and visually distinguishable digits would be easier to identify. The identification of digits based on visual features occurs in two stages - first the specific digit shape (allograph) and then the abstract digit identity (McCloskey \& Schubert, 2014). In Fig. 8, the identification of allographs would lie somewhere between the visual feature identification and the digit detectors.

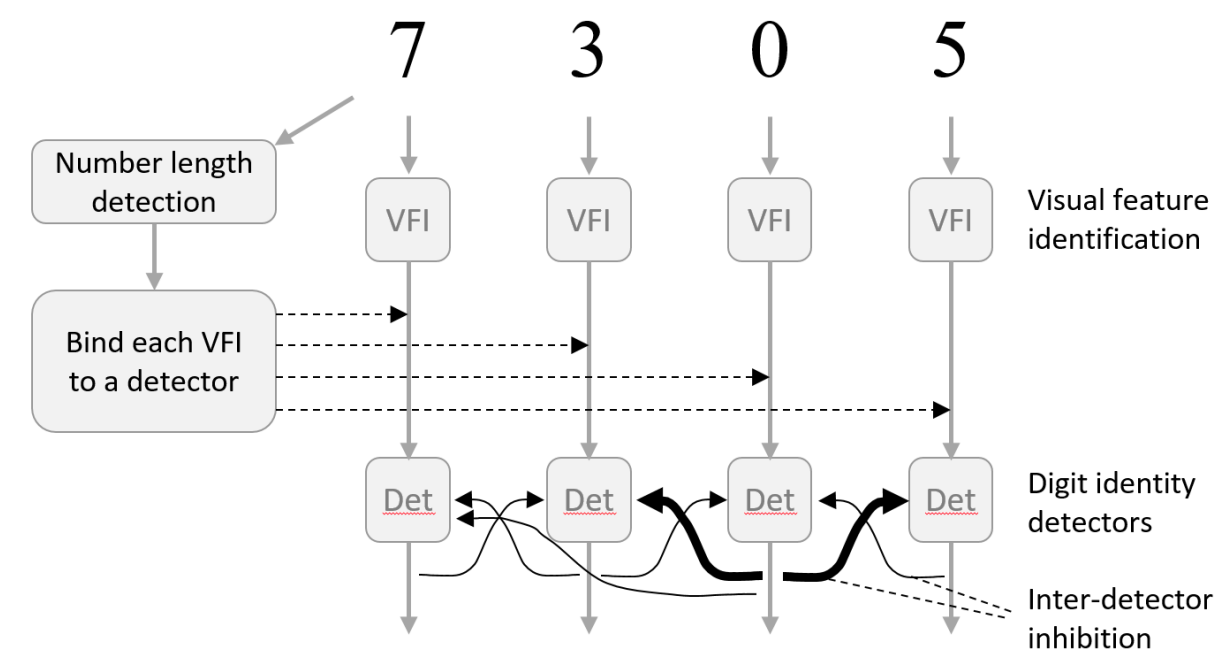

Fig. 8. Proposed model for the visual analysis of digit strings. The top layer is the initial analysis of the visual information; this analysis ends with the identification of character-parts typical to digits and letters (visual features). Each of the visual channels - one per digit - is bound (possibly in a serial manner) to a digit detector whose role is to identify the digit in a particular decimal position. Correct binding depends on knowing the number length, which, in turn, depends on detecting the leftmost digit. The distinction between the visual-feature channels is imperfect, so visual information of each digit leaks to adjacent channels. To cope with these leaks, the detectors are connected in inhibitory relations: the output of each detector inhibits the identification of the same digit by other detectors. The inhibition from the detectors of 0 and 1 is stronger (thicker arrows) than the inhibition from the detectors of 2-9, and the inhibition of adjacent digit is stronger than far digits. With minor modifications, this model can be adapted to an architecture in which there are 10 detectors, one for each of the digits $0-9$, rather than one detector per decimal position.

The array of digit detectors should be configured according to the number of digits in the number and their retinal positions (Davis, 2010; Dotan \& Dehaene, 2020b). This configuration could, for example, prepare the appropriate number of active detectors, bind each detector to the relevant input channel of visual features, and perhaps bind each detector to a relevant output channel. The detectors' output is the identified digits; the specific destination of this output depends on the task at hand. In our case, the task was reading aloud, so the detectors' output should be sent to the verbal number production processes. The binding process described here may overlap the process to which Dotan and Dehaene (2020b) refer as "syntactic frame creation". Importantly, for this binding to initiate, we must know how many digits the number has. This 
information is extracted by a dedicated process (Dotan \& Friedmann, 2018), which precedes the digit identification and may not distinguish between letters and digits (Dotan \& Dehaene, 2020b; McCloskey \& Schubert, 2014).

The detectors can operate in parallel, but a bottleneck process imposes seriality. This seriality could arise from the binding process described above, as it may bind the detectors serially. Alternatively, it could arise from the detection mechanism itself, if the detectors operate serially (Whitney, 2001), or from a process that reads the detectors' output serially from left to right (Perry et al., 2007; Wolford, 1975). The degree of seriality depends on the number length; for 2-digit and 3-digit numbers, it is negligible or nonexistent (Bahnmueller et al., 2016; Dehaene et al., 1990; Dotan \& Dehaene, 2020b; Moeller et al., 2009; Nuerk \& Willmes, 2005), but for longer numbers, a clear serial pattern emerges, as seen here and as previously reported by others (Friedmann et al., 2010; Meyerhoff et al., 2012).

The model assumes the detectors are inter-connected in inhibitory relations, whereby the identification of a digit in a particular position (a detector's output) inhibits the identification of this digit in other positions. Crucially, this lateral inhibition is stronger when the digit identified is 0 or 1 than when it is 2-9. The inhibition is also stronger for adjacent than nonadjacent detectors. Together, these two characteristics explain our finding that 0 and 1 facilitated the identification of adjacent digits more than nonadjacent digits. These inhibitory relations are the mechanism underlying the "zero detection" process hypothesized by Dotan and Friedmann (2018). Our results indicate that this process detects not only the positions of 0 but also the positions of 1 . Future studies may examine whether the visual analyzer treats 1 in a special manner independently of the task at hand or, as Dotan and Friedmann (2018) propose, only when the task involves verbal output, as this provides clear motivation to identify 1 because of its syntactic role in the verbal number system.

Our model assumes the special treatment of 0 and 1 is not at the level of digit detectors. It only occurs when the output of these detectors is sent to the next stage. This assumption is in excellent agreement with the conclusions of Schubert et al. (2020). They described a patient with a selective deficit in identifying the digits 2-9 and spared processing of 0-1. Interestingly, although this patient could not identify 2-9 consciously, his ERP signals still showed the digits were identified at some pre-conscious stage. Schubert et al. concluded the patient's deficit (the origin of his dissociation between 2-9 and 0-1) was not in the digit detection mechanism itself, but in "the signals sent from digit-identification processes". Correspondingly, our model attributes the special treatment of 0 and 1 precisely to these signals sent from the digit detectors.

The model can be easily adapted to an architecture in which there is one detector for each of the 10 possible digits (i.e., 10 detectors; see Davis, 2010; Whitney, 2001) rather than one detector per decimal position. Such an architecture would require two minor modifications in the model. Importantly, neither modification concerns the role of any of the cognitive mechanisms proposed 
above, only the way they are implemented. First, the initial configuration of detectors according to the number of stimulus digits may take a different form. For example, if digit position is encoded as the detector's activity pattern, such that each position yields a different pattern, it is possible that the detectors would be configured according to the number of different activity patterns expected for the specific stimulus length. Second, the mechanism that inhibits the leakage of a digit's identity to nearby positions would be implemented in a different manner under this architecture. Once a detector identifies the digit position, the encoding of the same position is inhibited in all other detectors. The inhibitory connections originating in the detectors of 0 and 1 would be stronger than those originating in the detectors of 2-9.

\subsection{Why are there separate visual analyzers for words and numbers?}

Several studies show that digit strings are handled by dedicated cognitive processes, separate from the mechanisms handling letters and words (Crutch \& Warrington, 2007; Déjerine, 1892; Dotan \& Friedmann, 2019; Friedmann \& Nachman-Katz, 2004; Nachman-Katz \& Friedmann, 2008; Priftis et al., 2013). The visual analyzers of digit strings and letter strings also reside in separate brain regions (Abboud et al., 2015; Baker et al., 2007; Grotheer et al., 2016; Hannagan et al., 2015; Park et al., 2012; Shum et al., 2013). Dotan and Friedmann (2019) suggested that the reason for this separation lies in the different syntactic structure of digit and letter strings, which therefore need to be parsed by different cognitive mechanisms. In other words, the different syntactic properties of numbers and words create a qualitative difference between the visual analyzer of numbers and the visual analyzer of words, even if the identification of a single letter and a single digit could have been perhaps carried out by a single process. Our study offers evidence supporting this idea. We showed that the visual analysis of digit strings is adapted to specific properties of numbers: the special role of 0 and 1 , and the emphasis on the initial digits (presumably because the initial digits are pronounced first when saying the number and have a greater magnitude as quantities). Notably, if the number-specific syntactic mechanism, the process detecting the positions of 0 and 1, is implemented as our model proposes, it does not seem like a mechanism that could easily have a neural implementation separate from the digit identification. Rather, it seems an intrinsic part of the digit identification process. Under this kind of architecture, it could be quite hard for a single visual analyzer to accommodate the different syntactic structures of numbers and words.

An additional explanation of the separation between the visual analysis of words and numbers comes from studies proposing that the visual analysis of words has developed to employ brain regions with high connectivity to language areas, whereas the visual analysis of numbers has developed to employ brain regions with high connectivity to quantity-processing areas (Abboud et al., 2015; Hannagan et al., 2015). According to this view, the distinction between numbers and words originates in the different destinations of the analyzed information. Note that this interpretation and ours are not mutually exclusive but complementary. Future studies might 
examine if and how these two motivations have interacted to create the two separate and specialized visual analysis mechanisms in the brain.

\section{References}

Abboud, S., Maidenbaum, S., Dehaene, S., \& Amedi, A. (2015). A number-form area in the blind. Nature Communications, 6, 6026. https://doi.org/10.1038/ncomms7026

Bahnmueller, J., Huber, S., Nuerk, H. C., Göbel, S. M., \& Moeller, K. (2016). Processing multi-digit numbers: a translingual eye-tracking study. Psychological Research, 80(3), 422-433. https://doi.org/10.1007/s00426-0150729-y

Baker, C. I., Liu, J., Wald, L. L., Kwong, K. K., Benner, T., \& Kanwisher, N. (2007). Visual word processing and experiential origins of functional selectivity in human extrastriate cortex. Proceedings of the National Academy of Sciences of the United States of America, 104(21), 9087-9092. https://doi.org/10.1073/pnas.0703300104

Bates, D., Mächler, M., Bolker, B., \& Walker, S. (2015). Fitting linear mixed-effects models using lme4. Journal of Statistical Software, 67(1), 1-48. https://doi.org/10.18637/jss.v067.i01

Benson, D. F., \& Denckla, M. B. (1969). Verbal paraphasia as a source of calculation disturbance. Archives of Neurology, 21(1), 96-102. https://doi.org/10.1001/archneur.1969.00480130110011

Bouma, H. (1973). Visual interference in the parafoveal recognition of initial and final letters of words. Vision Research, 13(4), 767-782. https://doi.org/https://doi.org/10.1016/0042-6989(73)90041-2

Chanceaux, M., \& Grainger, J. (2012). Serial position effects in the identification of letters, digits, symbols, and shapes in peripheral vision. Acta Psychologica, 141(2), 149-158. https://doi.org/https://doi.org/10.1016/j.actpsy.2012.08.001

Cohen, L., \& Dehaene, S. (1991). Neglect dyslexia for numbers? A case report. Cognitive Neuropsychology, 8(1), 39-58. https://doi.org/10.1080/02643299108253366

Crutch, S. J., \& Warrington, E. K. (2007). Word form access dyslexia: Understanding the basis of visual reading errors. The Quarterly Journal of Experimental Psychology, 60(1), 57-78. https://doi.org/10.1080/17470210600598676

Davis, C. J. (2010). The spatial coding model of visual word identification. In Psychological Review (Vol. 117, Issue 3, pp. 713-758). American Psychological Association. https://doi.org/10.1037/a0019738

Dehaene, S. (1992). Varieties of numerical abilities. Cognition, 44(1-2), 1-42. https://doi.org/10.1016/00100277(92)90049-N

Dehaene, S. (1997). The number sense: How the mind creates mathematics. Oxford University Press.

Dehaene, S. (2007). Symbols and quantities in parietal cortex: Elements of a mathematical theory of number representation and manipulation. In P. Haggard, Y. Rossetti, \& M. Kawato (Eds.), Attention and Performance, vol. 22: Sensorimotor foundations of higher cognition (pp. 527-574). Harvard University Press. https://doi.org/10.1093/acprof:oso/9780199231447.003.0024

Dehaene, S., Cohen, L., Sigman, M., \& Vinckier, F. (2005). The neural code for written words: a proposal. Trends in Cognitive Sciences, 9(7), 335-341. https://doi.org/10.1016/j.tics.2005.05.004

Dehaene, S., Dupoux, E., \& Mehler, J. (1990). Is numerical comparison digital? Analogical and symbolic effects in two-digit number comparison. Journal of Experimental Psychology: Human Perception and Performance, 16(3), 626-641.

Dehaene, S., \& Mehler, J. (1992). Cross-linguistic regularities in the frequency of number words. Cognition, 43(1), 1-29. https://doi.org/10.1016/0010-0277(92)90030-L

Déjerine, J. (1892). Contribution à l'étude anatomo-pathologique et clinique des différentes variétés de cécité verbale. Mémoires de La Société de Biologie, 4, 61-90.

Dotan, D., \& Dehaene, S. (2016). On the origins of logarithmic number-to-position mapping. Psychological Review, 123(6), 637-666. https://doi.org/10.1037/rev0000038

Dotan, D., \& Dehaene, S. (2020a). Tracking priors and their replacement: Mental dynamics of decision making in the number-line task. PsyArXiv. https://doi.org/10.31234/osf.io/skcg4

Dotan, D., \& Dehaene, S. (2020b). Parallel and serial processes in number-to-quantity conversion. Cognition, 204, 
104387. https://doi.org/10.1016/j.cognition.2020.104387

Dotan, D., \& Friedmann, N. (2018). A cognitive model for multidigit number reading: Inferences from individuals with selective impairments. Cortex, 101, 249-281. https://doi.org/10.1016/j.cortex.2017.10.025

Dotan, D., \& Friedmann, N. (2019). Separate mechanisms for number reading and word reading: Evidence from selective impairments. Cortex, 114, 176-192. https://doi.org/10.1016/j.cortex.2018.05.010

Dotan, D., Friedmann, N., \& Dehaene, S. (2014). Breaking down number syntax: Spared comprehension of multidigit numbers in a patient with impaired digit-to-word conversion. Cortex, 59, 62-73.

https://doi.org/10.1016/j.cortex.2014.07.005

Drugowitsch, J., Wyart, V., Devauchelle, A. D., \& Koechlin, E. (2016). Computational precision of mental inference as critical source of human choice suboptimality. Neuron, 92(6), 1398-1411. https://doi.org/10.1016/j.neuron.2016.11.005

Estes, W. K., Allmeyer, D. H., \& Reder, S. M. (1976). Serial position functions for letter identification at brief and extended exposure durations. Perception \& Psychophysics, 19(1), 1-15. https://doi.org/10.3758/BF03199379

Friedmann, N., Dotan, D., \& Rahamim, E. (2010). Is the visual analyzer orthographic-specific? Reading words and numbers in letter position dyslexia. Cortex, 46(8), 982-1004. https://doi.org/10.1016/j.cortex.2009.08.007

Friedmann, N., \& Gvion, A. (2001). Letter position dyslexia. Cognitive Neuropsychology, 18(8), 673-696. https://doi.org/10.1080/02643290143000051

Friedmann, N., \& Nachman-Katz, I. (2004). Developmental neglect dyslexia in a Hebrew-reading child. Cortex, 40(2), 301-313.

Godwin, H. J., Hout, M. C., \& Menneer, T. (2014). Visual similarity is stronger than semantic similarity in guiding visual search for numbers. Psychonomic Bulletin \& Review, 21(3), 689-695. https://doi.org/10.3758/s13423013-0547-4

Gomez, P., Ratcliff, R., \& Perea, M. (2008). The overlap model: A model of letter position coding. In Psychological Review (Vol. 115, Issue 3, pp. 577-600). American Psychological Association. https://doi.org/10.1037/a0012667

Grotheer, M., Herrmann, K. H., \& Kovacs, G. (2016). Neuroimaging evidence of a bilateral representation for visually presented numbers. Journal of Neuroscience, 36(1), 88-97. https://doi.org/10.1523/JNEUROSCI.212915.2016

Hammond, E. J., \& Green, D. W. (1982). Detecting targets in letter and non-letter arrays. In Canadian Journal of Psychology/Revue canadienne de psychologie (Vol. 36, Issue 1, pp. 67-82). Canadian Psychological Association. https://doi.org/10.1037/h0081211

Hannagan, T., Amedi, A., Cohen, L., Dehaene-Lambertz, G., \& Dehaene, S. (2015). Origins of the specialization for letters and numbers in ventral occipitotemporal cortex. Trends in Cognitive Sciences, 19(7), 374-382. https://doi.org/10.1016/j.tics.2015.05.006

Kinoshita, S., \& Norris, D. (2009). Transposed-letter priming of prelexical orthographic representations. In Journal of Experimental Psychology: Learning, Memory, and Cognition (Vol. 35, Issue 1, pp. 1-18). American Psychological Association. https://doi.org/10.1037/a0014277

Kinoshita, S., Robidoux, S., Mills, L., \& Norris, D. (2014). Visual similarity effects on masked priming. Memory \& Cognition, 42(5), 821-833. https://doi.org/10.3758/s13421-013-0388-4

Ktori, M., Kingma, B., Hannagan, T., Holcomb, P. J., \& Grainger, J. (2014). On the time-course of adjacent and non-adjacent transposed-letter priming. Journal of Cognitive Psychology, 26(5), 491-505. https://doi.org/10.1080/20445911.2014.922092

McCloskey, M. (1992). Cognitive mechanisms in numerical processing: Evidence from acquired dyscalculia. Cognition, 44(1-2), 107-157. https://doi.org/10.1016/0010-0277(92)90052-J

McCloskey, M., \& Schubert, T. (2014). Shared versus separate processes for letter and digit identification. Cognitive Neuropsychology, 31(5-6), 437-460. https://doi.org/10.1080/02643294.2013.869202

McCloskey, M., Sokol, S. M., \& Goodman, R. A. (1986). Cognitive processes in verbal-number production: Inferences from the performance of brain-damaged subjects. Journal of Experimental Psychology: General, 115(4), 307-330. https://doi.org/10.1037/0096-3445.115.4.307

Meyerhoff, H. S., Moeller, K., Debus, K., \& Nuerk, H. C. (2012). Multi-digit number processing beyond the twodigit number range: A combination of sequential and parallel processes. Acta Psychologica, 140(1), 81-90. https://doi.org/10.1016/j.actpsy.2011.11.005 
Moeller, K., Fischer, M. H., Nuerk, H. C., \& Willmes, K. (2009). Sequential or parallel decomposed processing of two-digit numbers? Evidence from eye-tracking. The Quarterly Journal of Experimental Psychology, 62(2), 323-334. https://doi.org/10.1080/17470210801946740

Nachman-Katz, I., \& Friedmann, N. (2008). Developmental neglect dyslexia and its effect on number reading. Language and Brain, 7, 83-96 (in Hebrew).

Nuerk, H. C., \& Willmes, K. (2005). On the magnitude representations of two-digit numbers. Psychology Science, $47(1), 52-72$.

Park, J., Hebrank, A., Polk, T. A., \& Park, D. C. (2012). Neural dissociation of number from letter recognition and its relationship to parietal numerical processing. Journal of Cognitive Neuroscience, 24(1), 39-50. https://doi.org/10.1162/jocn_a_00085

Perea, M., Duñabeitia, J. A., \& Carreiras, M. (2008). Transposed-letter priming effects for close versus distant transpositions. Experimental Psychology, 55(6), 384-393.

Perry, C., Ziegler, J. C., \& Zorzi, M. (2007). Nested incremental modeling in the development of computational theories: The CDP+ model of reading aloud. In Psychological Review (Vol. 114, Issue 2, pp. 273-315). American Psychological Association. https://doi.org/10.1037/0033-295X.114.2.273

Pinhas, M., \& Tzelgov, J. (2012). Expanding on the mental number line: Zero is perceived as the "smallest". Journal of Experimental Psychology: Learning, Memory, and Cognition, 38(5), 1187-1205. https://doi.org/10.1037/a0027390

Priftis, K., Albanese, S., Meneghello, F., \& Pitteri, M. (2013). Pure left neglect for Arabic numerals. Brain and Cognition, 81(1), 118-123. https://doi.org/10.1016/j.bandc.2012.09.008

R Core Team. (2019). R: A language and environment for statistical computing. R Foundation for Statistical Computing. http://www.r-project.org

Rumelhart, D. E., \& McClelland, J. L. (1982). An interactive activation model of context effects in letter perception: II. The contextual enhancement effect and some tests and extensions of the model. In Psychological Review (Vol. 89, Issue 1, pp. 60-94). American Psychological Association. https://doi.org/10.1037/0033-295X.89.1.60

Schubert, T. M., Rothlein, D., Brothers, T., Coderre, E. L., Ledoux, K., Gordon, B., \& McCloskey, M. (2020). Lack of awareness despite complex visual processing: Evidence from event-related potentials in a case of selective metamorphopsia. Proceedings of the National Academy of Sciences, 117(27), 16055-16064. https://doi.org/10.1073/pnas.2000424117

Shum, J., Hermes, D., Foster, B. L., Dastjerdi, M., Rangarajan, V., Winawer, J., Miller, K. J., \& Parvizi, J. (2013). A brain area for visual numerals. Journal of Neuroscience, 33(16), 6709-6715. https://doi.org/10.1523/JNEUROSCI.4558-12.2013

Townsend, J. T., Taylor, S. G., \& Brown, D. R. (1971). Lateral masking for letters with unlimited viewing time. Perception \& Psychophysics, 10(5), 375-378. https://doi.org/10.3758/BF03207464

Tydgat, I., \& Grainger, J. (2009). Serial position effects in the identification of letters, digits, and symbols. In Journal of Experimental Psychology: Human Perception and Performance (Vol. 35, Issue 2, pp. 480-498). American Psychological Association. https://doi.org/10.1037/a0013027

Whitney, C. (2001). How the brain encodes the order of letters in a printed word: The SERIOL model and selective literature review. Psychonomic Bulletin \& Review, 8(2), 221-243. https://doi.org/10.3758/BF03196158

Wolford, G. (1975). Perturbation model for letter identification. In Psychological Review (Vol. 82, Issue 3, pp. 184199). American Psychological Association. https://doi.org/10.1037/0033-295X.82.3.184

Wolford, G., \& Hollingsworth, S. (1974a). Evidence that short-term memory is not the limiting factor in the tachistoscopic full-report procedure. Memory \& Cognition, 2(4), 796-800. https://doi.org/10.3758/BF03198158

Wolford, G., \& Hollingsworth, S. (1974b). Lateral masking in visual information processing. Perception \& Psychophysics, 16(2), 315-320. https://doi.org/10.3758/BF03203949 